\title{
Entanglement in coupled kicked tops with chaotic dynamics
}

\author{
Tabea Herrmann, ${ }^{1}$ Maximilian F. I. Kieler, ${ }^{1}$ Felix Fritzsch, ${ }^{1}$ and Arnd Bäcker ${ }^{1,2}$ \\ ${ }^{1}$ Technische Universität Dresden, Institut für Theoretische Physik and Center for Dynamics, 01062 Dresden, Germany \\ ${ }^{2}$ Max-Planck-Institut für Physik komplexer Systeme, Nöthnitzer Straße 38, 01187 Dresden, Germany
}

(Dated: October 31, 2019)

\begin{abstract}
The entanglement of eigenstates in two coupled, classically chaotic kicked tops is studied in dependence of their interaction strength. The transition from the non-interacting and unentangled system towards full random matrix behavior is governed by a universal scaling parameter. Using suitable random matrix transition ensembles we express this transition parameter as a function of the subsystem sizes and the coupling strength for both unitary and orthogonal symmetry classes. The universality is confirmed for the level spacing statistics of the coupled kicked tops and a perturbative description is in good agreement with numerical results. The statistics of Schmidt eigenvalues and entanglement entropies of eigenstates is found to follow a universal scaling as well. Remarkably this is not only the case for large subsystems of equal size but also if one of them is much smaller. For the entanglement entropies a perturbative description is obtained, which can be extended to large couplings and provides very good agreement with numerical results. Furthermore, the transition of the statistics of the entanglement spectrum towards the random matrix limit is demonstrated for different ratios of the subsystem sizes.
\end{abstract}

\section{INTRODUCTION}

Entanglement is one of the key features of quantum theory and, besides of being of fundamental conceptual interest [1], has nowadays many applications ranging from quantum communication, quantum cryptography, to quantum computing [2-4]. It also plays an important role in characterizing phases of quantum many-body systems [5-11]. A fundamental condition for entanglement is a system consisting of multiple interacting subsystems. The simplest model to investigate entanglement properties of such systems are bipartite systems, which consist of two subsystems coupled by some interaction. One of the central questions for such bipartite systems concerns the possible amount of entanglement, quantified e.g. by the von Neumann entropy, Rényi entropies, Havrda-CharvátTsallis (HCT) entropies, or the Schmidt eigenvalues [12 16]. This question concerns both the entanglement generated in the time-evolution of initially un-entangled states and the entanglement of eigenstates of the full system. A common scenario is that the subsystems are "quantum-chaotic" in the sense that their spectral statistics and eigenstate statistics are welldescribed by random matrix theory. Such systems could have a classical limit with chaotic dynamics, while in the context of many-body systems a classical limit not necessarily exists.

If the subsystems are strongly coupled, their bipartite entanglement can be obtained from a random matrix description. This implies that the statistics of Schmidt eigenvalues is given by the Marčenko-Pastur distribution [17, 18] and leads to predictions for the average values of the purity (or linear entropy) [19] and von Neumann entropy [20, 21]. These results apply for example to quantum systems with classically chaotic dynamics, as shown for coupled standard maps [22] or coupled kicked tops [23-25], and to chaotic states in many body systems, see e.g. Refs. [26-31].

If the subsystems are not strongly interacting, the amount of eigenstate entanglement is reduced. For bipartite systems with broken time-reversal symmetry this has been intensively studied in the last few years [32-34]: a universal transition from unentangled to entangled states was found to be deter- mined by a single transition parameter depending only on the system sizes and the interaction strength. Furthermore a random matrix transition ensemble was introduced which allows to describe the universal features of entanglement and spectral statistics. Moreover a perturbation theoretical description for spectral statistics (consecutive level spacing distribution) and different measures of entanglement has been developed. For the entanglement entropies a recursively applied embedded perturbation theory describes the whole transition towards maximal entanglement [33, 34]. Recently, a perturbative description of the time-dependence of entanglement entropies for initial product eigenstates was obtained which leads to a universal prediction after an appropriate rescaling of time [35].

In this paper we study eigenstate entanglement in bipartite systems with and without time reversal symmetry and different types of couplings between the subsystems, based on techniques developed in Refs. [32-34]. To illustrate the analytical results we use a pair of coupled kicked tops as a system with time reversal invariance for both equal and different subsystem dimensions. The kicked top model was set up to study the influence of classical regular and chaotic behavior on quantum mechanical properties [36-38]. Coupled kicked tops have been introduced to investigate the time evolution of entanglement [39], and since then explored in much detail, see e.g. Refs. [23, 25, 39. 49]. Kicked tops are of particular interest, as they can also be accomplished experimentally [50-54] and realizing coupled kicked tops might therefore be feasible in the future. This would also provide a possibility to probe entanglement in a coupled many-body system as the total spin of each subsystem can be considered as the sum of spin-1/2 qubits [55-58]. We study the eigenstate entanglement for coupled kicked tops when both show fully chaotic behavior in the classical limit. For this we derive the transition parameter $\Lambda$ for the general case of systems with time reversal invariance and specifically for the random matrix transition ensemble with random diagonal coupling. To account for the specific interaction of the coupled kicked tops it turned out to be necessary to introduce a random matrix transition ensem- 
ble with random product phases for the coupling. Furthermore we develop a perturbation theory of the level spacing statistics for same subsystem dimensions and find a prediction for the uncoupled situation for different subsystem dimensions. To describe the entanglement of the coupled kicked tops in dependence on the transition parameter, we use a perturbative description for the first two Schmidt eigenvalues and for the entanglement entropies. Applying the recursive embedding of the regularized perturbation theory, following Ref. [34], leads to a description of the complete transition. Good agreement with numerical calculations for same as well as for different subsystem dimension is found. In addition we show that the distribution of the Schmidt eigenvalues approaches the Marčenko-Pastur distribution for large transition parameters, even though quite slowly.

The paper is organized as follows: In Sec. II we introduce bipartite systems and their time evolution operator and in Sec. II A define the transition parameter for which a general expression is obtained if the individual subsystems can be described by random matrix theory. Section II B discusses different random matrix transition ensembles with their transition parameters and statistical properties. In Sec. IIC we introduce the coupled kicked tops and the transition parameter for this system. In Sec. III the level spacing statistics is studied and a perturbative description is derived for the case of equal subsystem dimension and also the case of different subsystem dimensions is considered. Using the level spacing distribution we demonstrate the universality of the transition parameter. In Sec. IV we study the entanglement and its perturbative description for coupled kicked tops. For this we introduce in Sec. IVA the Schmidt eigenvalues and the entanglement entropies as measures for the entanglement in bipartite systems. In Sec. IVB we present perturbation theory results for the first two Schmidt eigenvalues. This perturbation theory is extended in Sec. IV C to the entanglement entropies and the recursively embedded perturbation theory is employed to describe the whole transition. In Sec. IVD we discuss the applicability of this theory to the case of different subsystem dimensions and in Sec. IVE the full statistics of the Schmidt eigenvalues is considered. Finally, a summary and outlook is given in Sec. V

\section{BIPARTITE SYSTEMS}

We consider a class of interacting bipartite systems in which the time evolution is given by a unitary Floquet operator, i.e. the propagator over one period of the driving, as

$$
\mathcal{U}=U_{12}(\varepsilon)\left(U_{1} \otimes U_{2}\right) .
$$

Here $U_{1}$ and $U_{2}$ are unitary operators on Hilbert spaces of dimension $N_{1}$ and $N_{2}$, respectively, and $U_{12}(\varepsilon)$ acts on the tensor product space of dimension $N_{1} N_{2}$ and provides a coupling between the two subsystems. The coupling is assumed to fulfill $U_{12}(0)=\mathrm{Id}$, i.e. there is no interaction between the subsystems for $\varepsilon=0$. With increasing $\varepsilon$ the interaction increases and the operator $U_{12}(\varepsilon)$ is assumed to be entangling
[59, 60]. Its eigenvalue problem is given by

$$
\mathcal{U}\left|\psi_{n}\right\rangle=\exp \left(\mathrm{i} \varphi_{n}\right)\left|\psi_{n}\right\rangle
$$

with eigenstates $\left|\psi_{n}\right\rangle$ and corresponding eigenvalues $\exp \left(\mathrm{i} \varphi_{n}\right)$, which lie on the unit circle due to the unitarity of $\mathcal{U}$, so that the eigenphases $\varphi_{n} \in[0,2 \pi[$. We aim to characterize the statistics of eigenphases and eigenstates in dependence on the strength $\varepsilon$ of the coupling and the Hilbert space dimensions $N_{1}$ and $N_{2}$.

\section{A. Universal transition parameter}

In various cases the statistical properties of the bipartite system (1) turn out to be governed by a single scaling parameter $\Lambda$ [32, 35, 61, 62]. This universal transition parameter is given by

$$
\Lambda=\frac{v^{2}}{D^{2}}
$$

where $v^{2}$ is the mean square off-diagonal matrix element of $U_{12}(\varepsilon)$ in the basis in which $U_{1} \otimes U_{2}$ is diagonal and $D=$ $\frac{2 \pi}{N_{1} N_{2}}$ is the mean level spacing of the full system.

For systems in which the non-interacting subsystems $U_{1}$ and $U_{2}$ can be modeled by random unitary matrices chosen from an appropriate ensemble the transition parameter depends on the coupling $U_{12}$ only. Specifically, in the absence of anti-unitary symmetries $U_{1}$ and $U_{2}$ are chosen from the circular unitary ensemble (CUE) while the circular orthogonal ensemble (COE) applies in the presence of an anti-unitary symmetry (e.g. time-reversal) [63].

The ensemble average for the COE leads to

$$
\begin{aligned}
\Lambda_{\mathrm{COE}}= & \frac{N_{1} N_{2}}{4 \pi^{2}\left(N_{1} N_{2}-1\right)\left(N_{1}+2\right)\left(N_{2}+2\right)} \\
& \times\left(N_{1} N_{2}\left(N_{1} N_{2}+2\left(N_{1}+N_{2}\right)\right)\right. \\
& \left.-2|| U_{12}^{(1)}\left\|^{2}-2\right\| U_{12}^{(2)} \|^{2}-\left|\operatorname{tr}\left(U_{12}\right)\right|^{2}\right),
\end{aligned}
$$

which is derived in App. A 1 . Here $U_{12}^{(1)}$ and $U_{12}^{(2)}$ are diagonal matrices with entries $\left(U_{12}^{(1)}\right)_{j j}=\sum_{k}\left(U_{12}\right)_{j k, j k}$, and $\left(U_{12}^{(2)}\right)_{k k}=\sum_{j}\left(U_{12}\right)_{j k, j k}$ as partially traced interaction operators, which are in general not unitary, and $\|X\|^{2}=\operatorname{Tr}\left(X X^{\dagger}\right)$ is the Hilbert-Schmidt norm [32, 34].

If the subsystems have equal dimension, $N=N_{1}=N_{2}$, Eq. (4) simplifies to

$$
\begin{aligned}
\Lambda_{\mathrm{COE}}=\frac{N^{4}}{4 \pi^{2}\left(N^{2}-1\right)(N+2)^{2}}\left(N^{2}+4 N\right. \\
\left.-2\left\|\frac{U_{12}^{(1)}}{N}\right\|^{2}-2\left\|\frac{U_{12}^{(2)}}{N}\right\|^{2}-\left|\frac{\operatorname{tr}\left(U_{12}\right)}{N}\right|^{2}\right) .
\end{aligned}
$$


For the CUE one gets

$$
\begin{aligned}
& \Lambda_{\mathrm{CUE}}=\frac{N_{1} N_{2}}{4 \pi^{2}\left(N_{1} N_{2}-1\right)\left(N_{1}+1\right)\left(N_{2}+1\right)} \\
& \times\left(N_{1} N_{2}\left(N_{1} N_{2}+\left(N_{1}+N_{2}\right)\right)\right. \\
& \left.-|| U_{12}^{(1)}\left\|^{2}-\right\| U_{12}^{(2)} \|^{2}-\left|\operatorname{tr}\left(U_{12}\right)\right|^{2}\right),
\end{aligned}
$$

which is derived in App. A2 For $N=N_{1}=N_{2}$ this simplifies to

$$
\begin{aligned}
\Lambda_{\mathrm{CUE}}=\frac{N^{4}}{4 \pi^{2}\left(N^{2}-1\right)(N+1)^{2}}\left(N^{2}+2 N\right. \\
\left.\quad-\left\|\frac{U_{12}^{(1)}}{N}\right\|^{2}-\left\|\frac{U_{12}^{(2)}}{N}\right\|^{2}-\left|\frac{\operatorname{tr}\left(U_{12}\right)}{N}\right|^{2}\right) .
\end{aligned}
$$

Note, that Eq. (7) differs slightly from the result obtained in Refs. [32, 34], but agrees in leading order for example with the results of the random matrix transition ensemble, see Eq. (13) below.

The above expressions for the transition parameter show that to obtain the same value of $\Lambda$ for different Hilbert space dimensions $N_{1}$ and $N_{2}$ the coupling strength $\varepsilon$ has to be adjusted accordingly. The explicit dependence on $\varepsilon$ is governed by the specific form of the coupling.

\section{B. Random matrix transition ensembles}

\section{General random matrix transition ensemble}

To define explicit random matrix models to describe the statistical properties of eigenvalues and eigenstates and the transition parameter of bipartite systems of the form (1) one has to prescribe the statistical properties of the coupling. The general form of the random matrix transition ensemble is

$$
\mathcal{U}_{\mathrm{RMT}}(\varepsilon)=U_{12}(\varepsilon)\left(U_{1}^{\mathrm{RMT}} \otimes U_{2}^{\mathrm{RMT}}\right),
$$

where $U_{1}^{\mathrm{RMT}}$ and $U_{2}^{\mathrm{RMT}}$ are random matrices, e.g. from the $\mathrm{COE}$ or the CUE. The coupling is written as

$$
U_{12}(\varepsilon)=\exp \left(\mathrm{i} \varepsilon V_{12}\right)
$$

and a rather general modeling is given by a diagonal matrix

$$
\left(V_{12}\right)_{j k, j^{\prime} k^{\prime}}=2 \pi \xi(j, k) \delta_{j j^{\prime}} \delta_{k k^{\prime}},
$$

with $j, j^{\prime}=1, \ldots, N_{1}$ and $k, k^{\prime}=1, \ldots, N_{2}$. The phase $\xi(j, k)$ is assumed to be random following some prescribed distribution.

\section{Random matrix transition ensemble}

In Ref. [32] the random matrix transition ensemble was introduced for which the coupling is given by

$$
\left(V_{12}\right)_{j k, j^{\prime} k^{\prime}}=2 \pi \xi_{j k} \delta_{j j^{\prime}} \delta_{k k^{\prime}},
$$

where $\xi_{j k}$ are i.i.d. distributed uniformly on $[-1 / 2,1 / 2]$. The limiting case of strong coupling has been studied in Ref. [64], where the entangling power of $\mathcal{U}_{\mathrm{CUE}}(\varepsilon=1)$ was derived analytically.

Using the general result (4) for the COE case gives, see App. A3

$$
\begin{aligned}
\Lambda_{\mathrm{COE}}= & \frac{N_{1}^{2} N_{2}^{2}}{4 \pi^{2}\left(N_{1}+2\right)\left(N_{2}+2\right)} \\
& \times \frac{\left(N_{1}+2\right)\left(N_{2}+2\right)-9}{N_{1} N_{2}-1}\left[1-\frac{\sin ^{2}(\pi \varepsilon)}{\pi^{2} \varepsilon^{2}}\right],
\end{aligned}
$$

and for the CUE,

$$
\begin{aligned}
\Lambda_{\mathrm{CUE}}= & \frac{N_{1}^{2} N_{2}^{2}}{4 \pi^{2}\left(N_{1}+1\right)\left(N_{2}+1\right)} \\
& \times \frac{\left(N_{1}+1\right)\left(N_{2}+1\right)-4}{N_{1} N_{2}-1}\left[1-\frac{\sin ^{2}(\pi \varepsilon)}{\pi^{2} \varepsilon^{2}}\right] .
\end{aligned}
$$

In the definition of the transition parameter (3) the offdiagonal elements of $U_{12}$ appear in the numerator. Thus, when applying the perturbation theory below to describe the spectral statistics and the entanglement in dependence on $\Lambda$, the distribution of the matrix elements

$$
\omega_{j k}=\frac{1}{\tilde{v}^{2}}\left|\left\langle j^{\prime} k^{\prime}\left|V_{12}\right| j k\right\rangle\right|^{2},
$$

in the eigenbasis $|j k\rangle$ of the uncoupled system plays an important role. Here $\tilde{v}$ is the mean square off-diagonal element of $V_{12}$ in this basis such that $\omega_{j k}$ has unit mean. For small $\varepsilon$ one has $U_{12}(\varepsilon)=\exp \left(\mathrm{i} \varepsilon V_{12}\right) \approx \mathrm{Id}+\mathrm{i} \varepsilon V_{12}$ and thus $v^{2}=\varepsilon^{2} \tilde{v}^{2}$. As there are no correlations between the matrix elements, the coupling (11) leads for the COE case to $\omega_{j k}$ following the Porter-Thomas distribution [65]

$$
\rho_{V_{12}}(\omega)=\frac{1}{\sqrt{2 \pi \omega}} \exp (-\omega / 2) .
$$

For the CUE transition ensemble one gets the exponential

$$
\rho_{V_{12}}(\omega)=\exp (-\omega)
$$

\section{Random matrix transition ensemble with product phases}

The coupling (11) provides the simplest possible form and leads to a good description of spectral statistics and entanglement in a wide class of systems [32-35]. However one may have other types of interactions leading to different expressions for the transition parameter and the statistical properties. A physically relevant case occurs when the matrix $V_{12}$ is the tensor product of matrices acting on the individual subsystems Hilbert spaces. In this case the phases can be described by a product of random individual phases,

$$
\left(V_{12}\right)_{j k, j^{\prime} k^{\prime}}=2 \pi \xi_{j} \tilde{\xi}_{k} \delta_{j j^{\prime}} \delta_{k k^{\prime}},
$$

where $\xi_{j}$ and $\tilde{\xi}_{k}$ are i.i.d. distributed uniformly on $[-1 / 2,1 / 2]$. Using the general result (4) for the COE case 
gives for small $\varepsilon$, see App. A 4

$$
\Lambda_{\mathrm{COE}} \simeq \frac{\varepsilon^{2}}{144} \frac{\left(N_{1} N_{2}\right)^{2}\left(\left(N_{1}+1\right)\left(N_{2}+1\right)-9\right)}{\left(N_{1} N_{2}-1\right)\left(N_{1}+2\right)\left(N_{2}+2\right)},
$$

and for the CUE

$$
\Lambda_{\mathrm{CUE}} \simeq \frac{\varepsilon^{2}}{144} \frac{\left(N_{1} N_{2}\right)^{2}\left(\left(N_{1}+1\right)\left(N_{2}+1\right)-4\right)}{\left(N_{1} N_{2}-1\right)\left(N_{1}+1\right)\left(N_{2}+1\right)} .
$$

The full expressions, valid for larger $\varepsilon$ as well, are also given in App. A 4

Moreover, due to the product structure of the phases, the distribution of the matrix elements $\omega_{j k}$ is given by

$$
\rho_{V_{12}}(\omega)=\frac{1}{\pi \sqrt{w}} K_{0}(\sqrt{w})
$$

see App. C. where $K_{0}$ is the modified Bessel function of the second kind [66, Eq. 10.25.3]. For the CUE case one gets

$$
\rho_{V_{12}}(\omega)=2 K_{0}(2 \sqrt{\omega})
$$

\section{Example: Coupled kicked tops}

As specific example of an interacting bipartite system we consider a pair of coupled time-periodically kicked tops, which have been studied in much detail in particular with respect to entanglement generation, see e.g. Refs. 23, 25, 39. 48]. The dynamics is described by the Hamiltonian [24, 39]

$$
H(t)=H_{1}(t)+H_{2}(t)+H_{12}(t),
$$

where

$$
\begin{aligned}
H_{\ell}(t) & =\frac{\pi}{2} J_{y_{\ell}}+\frac{k_{\ell}}{2 j_{\ell}}\left(J_{z_{\ell}}+\alpha_{\ell}\right)^{2} \sum_{n=-\infty}^{\infty} \delta(t-n), \\
H_{12} & =\varepsilon \frac{1}{\sqrt{j_{1} j_{2}}} J_{z_{1}} J_{z_{2}} \sum_{n=-\infty}^{\infty} \delta(t-n) .
\end{aligned}
$$

Here $j_{\ell}$ is the total angular momentum of the $\ell$-th $\operatorname{spin}(\ell=$ $1,2)$, and $J_{y_{\ell}}$ und $J_{z_{\ell}}$ are the components of the angular momentum operator. Although the following equally applies to half integer spins we for simplicity restrict the discussion to integer $j_{\ell}$. The parameters $k_{\ell}$ are the individual kicking strengths of the two tops and $\varepsilon$ determines the coupling strength between the two tops. For $\varepsilon=0$ the two subsystems are uncoupled. The Hilbert spaces of the uncoupled spins have dimension $N_{1}=2 j_{1}+1$ and $N_{2}=2 j_{2}+1$, respectively. The real parameters $\alpha_{\ell}$ are additional phases which allow to break the parity symmetry [24].

The Floquet operator for the coupled tops is given by [24]

$$
\mathcal{U}=U_{12}(\varepsilon)\left(U_{1} \otimes U_{2}\right)
$$

where

$$
U_{\ell}=\exp \left(-\frac{\mathrm{i} k_{\ell}}{2 j_{\ell}}\left(J_{z_{\ell}}+\alpha_{\ell}\right)^{2}\right) \exp \left(-\frac{\mathrm{i} \pi}{2} J_{y_{\ell}}\right),
$$

and the coupling reads

$$
U_{12}(\varepsilon)=\exp \left(\mathbf{i} \varepsilon V_{12}\right) \quad \text { with } V_{12}=\frac{1}{\sqrt{j_{1} j_{2}}} J_{z_{1}} J_{z_{2}}
$$

The order of the operators is such that we consider the free evolution first and then apply the kicks.

In the following we use $k_{1}=12.0$ and $k_{2}=15.0$ for which the classical dynamics corresponding to each top in the uncoupled case (numerically) shows chaotic motion with no visible regular structures. As phases we use $\alpha_{1}=0.35$ and $\alpha_{2}=0.4$ so that there is only time-reversal symmetry [24]. Therefore the Floquet operators $U_{\ell}$ for the individual spins and their spectral statistics can be modeled by the COE.

To compute the transition parameter for the coupled kicked tops we replace $U_{\ell}$ by independent COE matrices to use the general COE result (4) and compute the specific expressions for the coupling (27), see Eqs. (A22)-A24) in App. A 5. In the numerical computations these expressions are used to determine $\Lambda$ in dependence on $\varepsilon$ and $j_{\ell}$. For large $N_{1}, N_{2}$ and small $\varepsilon$ one gets

$$
\Lambda \approx \frac{1}{144 \pi^{2}} \varepsilon^{2} N_{1} N_{2}\left[N_{1} N_{2}+2\left(N_{1}+N_{2}\right)\right]
$$

As discussed before in Sec. II A, this expression explicitly shows that to get the same $\Lambda$ for different $j_{1}$ and $j_{2}$ one has to adapt the coupling accordingly.

\section{LEVEL SPACING STATISTICS}

\section{A. Level spacing statistics for equal dimensions}

To demonstrate that the transition parameter indeed leads to a universal description for the coupled kicked tops, we first consider the distribution of consecutive level spacings for equal Hilbert space dimensions $N=N_{1}=N_{2}$. The distribution $P(s)$ of the (re-scaled) consecutive level spacings $s_{n}=\frac{1}{D}\left(\varphi_{n+1}-\varphi_{n}\right)$, where $D$ is the mean level spacing, depends on the strength of the coupling between the subsystems: For strong coupling $P(s)$ should follow the results of the COE [67] which is well-described by the Wigner distribution

$$
P_{\mathrm{COE}}(s) \approx \frac{\pi}{2} s \exp \left(-\frac{\pi}{4} s^{2}\right) .
$$

For the uncoupled case, even though the individual subsystems show COE statistics, the resulting spacing distribution of the full bipartite system for large $N_{1}$ and $N_{2}$ approaches the exponential

$$
P_{\text {Poisson }}(s)=\exp (-s) \text {. }
$$

The reason for this is that the eigenphases of the full system (1) are an independent superposition $\varphi_{j k}=\theta_{j}^{(1)}+\theta_{k}^{(2)} \bmod 2 \pi$ of the phases $\theta_{j}^{(1)}$ and $\theta_{k}^{(2)}$ of the individual subsystems, respectively, where $j=1, \ldots, N_{1}$ and $k=1, \ldots, N_{2}$. Note that for tensor products of CUE matrices of equal dimension it has 

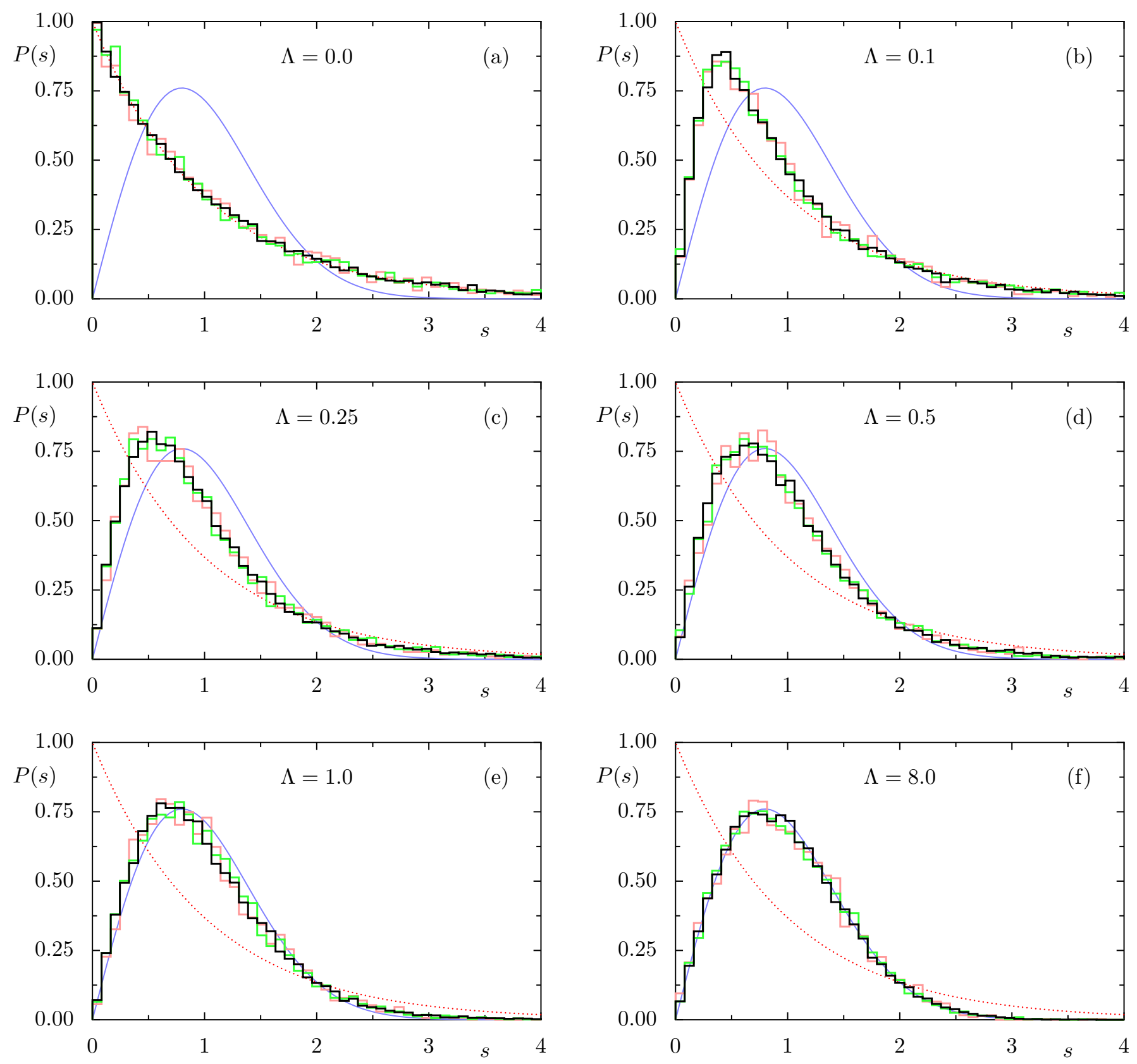

FIG. 1. Transition of the level spacing distribution for the coupled kicked tops for (a) $\Lambda=0.0$, (b) $\Lambda=0.1$, (c) $\Lambda=0.25$, (d) $\Lambda=0.5$, (e) $\Lambda=1.0$, and (f) $\Lambda=8.0$. The exponential (30), is shown as red dotted curve and the COE result (29), as blue solid curve. In each case the histograms for $j_{1}=j_{2}=30$ (very light red), $j_{1}=j_{2}=50$ (light green), and $j_{1}=j_{2}=70$ (black) are shown. The other parameters are $k_{1}=12.0, k_{2}=15.0, \alpha_{1}=0.35$, and $\alpha_{2}=0.4$.

been proven in Ref. [68] that the spectral statistics become Poissonian.

Figure 1 shows the level spacing distribution $P(s)$ for different $j_{1}=j_{2}=30,50,70$ in dependence on $\Lambda$. At $\Lambda=0$ one obtains good agreement with the exponential (30). Once $\Lambda>0$ there is an instantaneous change to level-repulsion, i.e. $P(0)=0$, as illustrated in Fig. 1 b) for $\Lambda=0.1$. Increasing $\Lambda$ further one gets closer to the result for the COE, Eq. (29). While the initial change of the distribution is rather rapid in $\Lambda$, this slows down at around $\Lambda=1.0$ and the COE statistics is well fulfilled at $\Lambda=8.0$, see Fig. 11ff). Interestingly, this happens significantly later than in case of the CUE transition ensemble and the coupled kicked rotors on the torus where good agreement is found at $\Lambda=1.0$ [32]. Numerical results for the transition ensemble (8) with COE matrices and interaction (11) show the same slower approach to the COE limit 29). Thus this is an inherent feature of the considered COE case and not specific to the coupling 27) of the kicked tops.

The sequence of plots in Fig. 1 confirms that $\Lambda$ provides the universal scaling parameter: For the same $\Lambda$, but different 


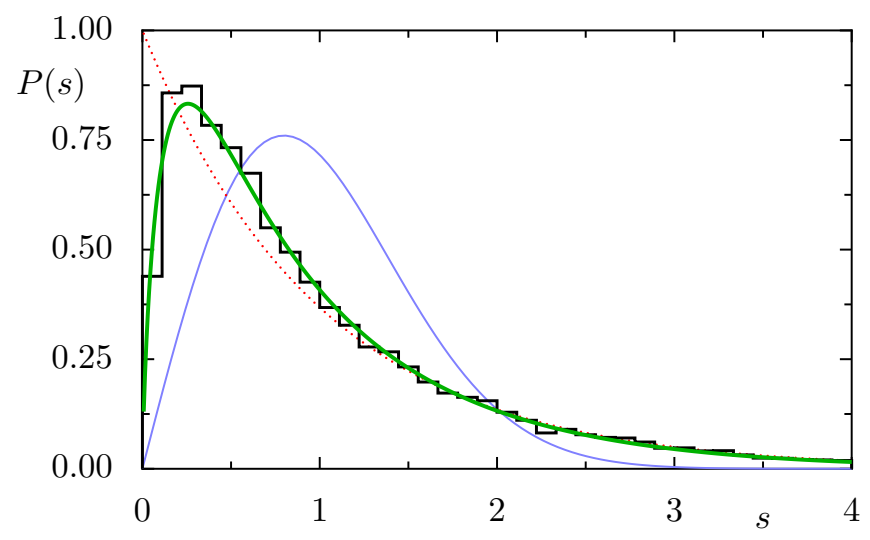

FIG. 2. Perturbative prediction of the level spacing distribution for the coupled kicked tops at small $\Lambda=0.02$. Shown is the histogram for $j_{1}=j_{2}=70, k_{1}=12.0, k_{2}=15.0, \alpha_{1}=0.35$, and $\alpha_{2}=0.4$. The thick green curve shows the result of the perturbation theory (38). For comparison the result 29] for the COE (solid blue curve), and the exponential 30 (red dotted curve) are shown.

$j_{1}=j_{2}$ and corresponding $\varepsilon$, determined implicitly using (4) and Eqs. A19- A21, the histograms nicely fall on top of each other.

In order to derive a perturbative expression for $P(s)$ we closely follow the derivation in the CUE case given in Ref. [34] and adapt this to the COE transition ensemble. We choose the specific interaction for the coupled kicked tops given by Eq. 27) for which $V_{12}$ is a tensor product of spin operators acting on the individual subsystems. Thus we model its statistics by a random interaction of product form defined in Eq. (17). The starting point is the perturbative expansion of the eigenphases $\varphi_{j k}$ of $\mathcal{U}$ according to [34]

$$
\varphi_{j k}=\theta_{j k}+\varepsilon\left\langle j k\left|V_{12}\right| j k\right\rangle+\varepsilon^{2} \sum_{j^{\prime} k^{\prime} \neq j k} \frac{\left|\left\langle j^{\prime} k^{\prime}\left|V_{12}\right| j k\right\rangle\right|^{2}}{\theta_{j k}-\theta_{j^{\prime} k^{\prime}}} .
$$

Here $\theta_{j k}$ are the eigenphases of the unperturbed system, i.e. for $\varepsilon=0$, corresponding to the eigenstates $|j k\rangle$, with $j=$ $1, \ldots, N_{1}$ and $k=1, \ldots ., N_{2}$, which form a basis of the full Hilbert space. To compute the distribution of the normalized consecutive level spacings

$$
s_{j k}=\frac{\varphi_{j k}-\varphi_{j^{1} k^{1}}}{D}
$$

where $\left|j^{1} k^{1}\right\rangle$ is the eigenstate for which $\theta_{j^{1} k^{1}}$ is the consecutive eigenphase of $\theta_{j k}$ in the unperturbed system we take the average over the random matrix transition ensemble with product phases, see Sec. III 3 Doing so not all terms in the perturbative expression contribute to the consecutive level spacing. In particular, the first order correction merely shifts the whole spectrum leaving all level spacings unchanged. Moreover, as the eigenphases $\theta_{j k}$ are uniformly distributed in $\left[0,2 \pi\left[\right.\right.$ only two second order terms $\sim \varepsilon^{2}$ contribute to the difference $\varphi_{j k}-\varphi_{j^{1} k^{1}}$ upon averaging. For simplicity we only keep these non-vanishing terms which leads to the level spacing [32]

$$
s_{j k}=\frac{\theta_{j k}-\theta_{j^{1} k^{1}}}{D}+2 \frac{\varepsilon^{2}}{D} \frac{\left|\left\langle j k\left|V_{12}\right| j^{1} k^{1}\right\rangle\right|^{2}}{\theta_{j k}-\theta_{j^{1} k^{1}}} .
$$

The averaging procedure is then performed by replacing $\left|\left\langle j k\left|V_{12}\right| j^{1} k^{1}\right\rangle\right|^{2}$ by $\tilde{v}^{2} \omega_{j k}$. Further $\left(\theta_{j k}-\theta_{j^{1} k^{1}}\right) / D$ is substituted by the spacing $s_{j k}^{(0)}$ in the unperturbed system. Noting that $v^{2}=\varepsilon^{2} \tilde{v}^{2}$ is the mean squared off-diagonal element of the full perturbation $\varepsilon V_{12}$ gives in lowest order of $\varepsilon$

$$
s_{j k}=s_{j k}^{(0)}+2 \Lambda \frac{\omega_{j k}}{s_{j k}^{(0)}}
$$

with $\Lambda$ as defined in Eq. (3).

In order to compute the statistics of the level spacings $s$ we average over both random variables $s_{j k}^{(0)}$ and $\omega_{j k}$. The distributions $P_{0}\left(s_{0}\right)$ of $s_{j k}^{(0)}$ is the Poisson distribution (29) and the distribution $\rho_{V_{12}}(\omega)$ of $\omega_{j k}$ is given by Eq. 20). The singular behavior of Eq. (34) at $s_{j k}^{(0)} \rightarrow 0$ is dealt with by regularization [32, 62, 69], i.e. the replacement

$$
s_{0}+2 \Lambda \frac{\omega}{s_{0}} \rightarrow \sqrt{s_{0}^{2}+4 \Lambda \omega} .
$$

This follows from degenerate perturbation theory and correctly captures the repulsion of nearly degenerate levels. Since this also provides the correct asymptotic behavior, this replacement can be done in the entire range of integration. The distribution of level spacing results in

$$
\begin{aligned}
\widetilde{P}(s)= & \int_{0}^{\infty} \mathrm{d} s_{0} \int_{0}^{\infty} \mathrm{d} \omega \rho_{V_{12}}(\omega) P_{0}\left(s_{0}\right) \\
& \times \delta\left(s-\sqrt{s_{0}^{2}+4 \Lambda \omega}\right) .
\end{aligned}
$$

Note, that due the regularization procedure $\widetilde{P}(s)$ does not have the required unit mean. This condition can be restored using a rescaling of $s$ by

$$
\overline{\mathbf{s}}=\int_{0}^{\infty} \mathrm{d} s s \widetilde{P}(s),
$$

such that the final result for the level spacing distribution reads

$$
P(s)=\overline{\mathbf{s}} \widetilde{P}(\overline{\mathbf{s}} s) .
$$

The resulting prediction as well as the level spacing distribution for the coupled kicked tops is shown in Fig. 2 for $\Lambda=0.02$. Here, we evaluate the integrals in Eq. (36) numerically. This figure demonstrates that there is a good agreement between the perturbation theory result and the data for the coupled kicked tops. Extending the prediction beyond the perturbative regime is an interesting open problem. Note that for obtaining the perturbative result (38) the specific form of the coupling (27) has been used. Using the random matrix transition ensemble with coupling given by Eq. (11) would lead to a prediction having its maximum further to the right. 


\section{B. Level spacing statistics for different dimensions}

The results shown in Fig. 1 and Fig. 2 confirm that the transition parameter provides a universal scaling in the case of equal Hilbert space dimensions $N_{1}=N_{2}$. Moreover, the above derivation allows to treat different dimensional subsystems, i.e. $N_{1} \neq N_{2}$, as well. In particular the case of $N_{1} \ll N_{2}$ is of relevance as it corresponds to one system with a small Hilbert space coupled to a system with chaotic dynamics and a much larger Hilbert space which could be considered as representing a heat-bath.

However, already for the uncoupled case, $\Lambda=0$, the level spacing statistics $P(s)$ shows clear deviations from the exponential behavior (30), see Fig. 3(a). This can be explained by the form $\varphi_{j k}=\theta_{j}^{(1)}+\theta_{k}^{(2)} \bmod 2 \pi$ of the eigenphases of the uncoupled system. If $N_{1} \ll N_{2}$, the statistics of the second subsystem is well described by the COE, so that the full spectrum can be considered as a superposition of $N_{1}$ independent COE ensembles of size $N_{2}$. In this case the consecutive level spacing distribution is given by the $N_{1} \mathrm{COE}$ statistics [70],

$$
P_{N_{1} \mathrm{COE}}(s)=t_{1}^{N_{1}-2} t_{2}\left[\left(1-\frac{1}{N_{1}}\right) t_{2}+\frac{\pi s}{2 N_{1}^{2}} t_{1}\right],
$$

where

$$
t_{1}=\operatorname{erfc}\left(\frac{\sqrt{\pi} s}{2 N_{1}}\right) \quad \text { and } \quad t_{2}=\exp \left(-\frac{\pi s^{2}}{4 N_{1}^{2}}\right),
$$

using the complementary error function. In Fig. 3 (3) we observe very good agreement of Eq. (39) with the numerical result for the small dimension $N_{1}=3$, corresponding to $j_{1}=1$. Furthermore we emphasise that Eq. (39) converges for $N_{1} \rightarrow \infty$ to the Poisson distribution (30). This is already rather well achieved for $N_{1}=11$, corresponding to $j_{1}=5$, which is also shown in Fig. 3. (a).

Thus the transition of the consecutive level spacing distribution cannot be universal if one subsystem dimension is small. There are significant differences for different $j_{1}$ which are also present when the coupling is increased. For strong coupling these differences disappear as the two subsystem merge into one large system, whose statistics becomes independent of the ratio of the subsystems sizes, and is given by that of the COE. We remark that the perturbation theory requires that both subsystem dimensions are large. Here we considered the extreme case that one dimension is small and thus it comes as no surprise that universality may fail.

\section{ENTANGLEMENT}

\section{A. Schmidt eigenvalues and entanglement entropies}

For the bipartite system (1) with tunable interaction the eigenstates of $\mathcal{U}(0)$, i.e. the uncoupled case, are simply product states of the individual subsystems and therefore not entangled. However with increasing interaction $\varepsilon$ they can no longer be written as product states, i.e. they become entangled. To characterize the amount of entanglement between
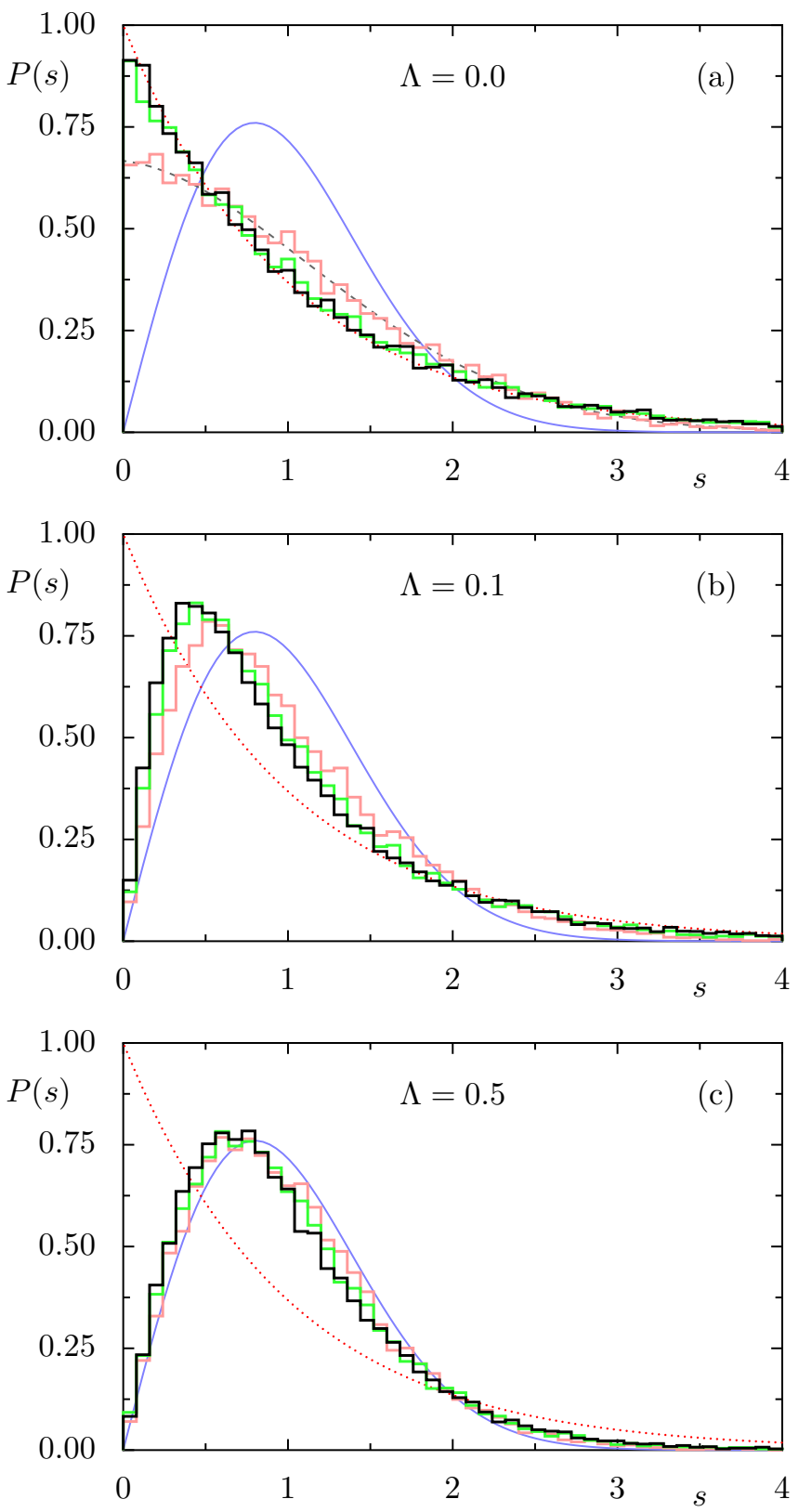

FIG. 3. Level spacing statistics for different dimensions of the two coupled kicked tops for (a) $\Lambda=0.0$, (b) $\Lambda=0.1$, and (c) $\Lambda=$ 0.5. The histograms are for $\left(j_{1}, j_{2}\right)=(1,3000)$, very light red, $\left(j_{1}, j_{2}\right)=(5,1000)$, light green, and $\left(j_{1}, j_{2}\right)=(10,500)$, black. The gray dashed curve in (a) shows the $N_{1} \mathrm{COE}$ statistics (39) for $N_{1}=3$. For comparison the result 29] for the COE (solid blue curve), and the exponential (30) (red dotted curve) are shown. The other parameters are $k_{1}=12.0, k_{2}=15.0, \alpha_{1}=0.35$, and $\alpha_{2}=$ 0.4 .

the two subsystems, there exist different quantitative measures, like the von Neumann entropy, Rényi entropies or the Havrda-Charvát-Tsallis entropies [14-16]. These measures are all based on the eigenvalues of the reduced density matrices for a given pure state $|\Phi\rangle$ of the full system,

$$
\rho_{1}=\operatorname{tr}_{2}(|\Phi\rangle\langle\Phi|), \quad \rho_{2}=\operatorname{tr}_{1}(|\Phi\rangle\langle\Phi|),
$$


which are defined as the partial trace over the other subsystem. For $N_{1} \leq N_{2}$ the reduced density matrices $\rho_{1}$ and $\rho_{2}$ have $N_{1}$ common eigenvalues $\lambda_{j}$, which are called Schmidt eigenvalues and obey the normalization condition

$$
\sum_{j=1}^{N_{1}} \lambda_{j}=1
$$

The remaining $N_{2}-N_{1}$ eigenvalues of $\rho_{2}$ are zero. In the following we assume the Schmidt eigenvalues to be ordered by decreasing value, i.e. $\lambda_{1} \geq \cdots \geq \lambda_{N_{1}}$. A state is unentangled if and only if $\lambda_{1}=1$ and all other Schmidt eigenvalues vanish. If $\lambda_{1}<1$, the state is entangled, as it is no longer represented as a product state. Maximal entanglement is obtained when $\lambda_{j}=1 / N_{1}$ for all $j=1, \ldots, N_{1}$.

Based on the Schmidt eigenvalues one can define the moments

$$
\mu_{\alpha}=\sum_{j=1}^{N_{1}} \lambda_{j}^{\alpha}, \quad \alpha>0
$$

and the Havrda-Charvát-Tsallis (HCT) entropies [14-16] by

$$
S_{\alpha}=\frac{1-\mu_{\alpha}}{\alpha-1}
$$

In the limit of $\alpha \rightarrow 1$ the von Neumann entropy $S_{1}$ is obtained,

$$
\begin{aligned}
S_{1} & =-\operatorname{tr}\left(\rho_{1} \ln \rho_{1}\right)=-\operatorname{tr}\left(\rho_{2} \ln \rho_{2}\right) \\
& =-\sum_{j=1}^{N_{1}} \lambda_{j} \ln \lambda_{j} .
\end{aligned}
$$

States which are unentangled lead to $S_{\alpha}=0$ while a maximally entangled state for example leads to $S_{1}=\ln N_{1}$.

For states chosen at random uniformly with respect to the Haar measure from the full Hilbert space, the average von Neumann entropy can be computed exactly [20, 21, 71, 72] and has the large $N_{1}$ asymptotics

$$
\overline{S_{1}}=\ln N_{1}-\frac{1}{2 N_{2} / N_{1}} .
$$

For the linear entropy $S_{2}$ the exact finite $-N_{1}$ result is [19]

$$
\overline{S_{2}}=1-\frac{N_{1}+N_{2}}{1+N_{1} N_{2}} .
$$

\section{B. Perturbative behavior of Schmidt eigenvalues}

As for the uncoupled bipartite system, Eq. (1) for $\varepsilon=0$, the states are not entangled, we have $\lambda_{1}=1$ and $\lambda_{j}=0$ for $j>1$. For non-vanishing coupling, the states become entangled such that $\lambda_{1}<1$ and the second largest Schmidt eigenvalue $\lambda_{2}$ gives the most relevant contribution. To arrive at a general expression for the first two averaged Schmidt eigenvalues we now follow Refs. [33, 34]. Based on RayleighSchrödinger perturbation theory it has been shown that the first two Schmidt eigenvalues can be approximated as

$$
\begin{aligned}
& \lambda_{1}^{j k}=1-\varepsilon^{2} \sum_{j^{\prime} k^{\prime} \neq j k} \frac{\left|\left\langle j k\left|V_{12}\right| j^{\prime} k^{\prime}\right\rangle\right|^{2}}{\left(\theta_{j k}-\theta_{j^{\prime} k^{\prime}}\right)^{2}}, \\
& \lambda_{2}^{j k}=\varepsilon^{2} \frac{\left|\left\langle j k\left|V_{12}\right| j^{2} k^{2}\right\rangle\right|^{2}}{\left(\theta_{j k}-\theta_{j^{2} k^{2}}\right)^{2}} .
\end{aligned}
$$

The notation is the same as introduced in Sec. III A Furthermore $\left|j^{2} k^{2}\right\rangle$ is the state for which $\theta_{j^{2} k^{2}}$ is closest to $\theta_{j k}$. Again $\left|\left\langle j k\left|V_{12}\right| j^{\prime} k^{\prime}\right\rangle\right|^{2}$ is replaced by $\tilde{v}^{2} \omega_{j^{\prime} k^{\prime}}$. In addition $\left(\theta_{j k}-\theta_{j^{\prime} k^{\prime}}\right) / D$ is substituted by $s_{j^{\prime} k^{\prime}}^{\mathrm{R}_{2}}$ and $\left(\theta_{j k}-\theta_{j^{2} k^{2}}\right) / D$ by the closer neighbor spacing $s_{j^{2} k^{2}}^{\mathrm{CN}}$. Using $v^{2}=\varepsilon^{2} \tilde{v}^{2}$ leads to

$$
\begin{aligned}
& \lambda_{1}^{j k} \approx 1-\Lambda \sum_{j^{\prime} k^{\prime} \neq j k} \frac{\omega_{j^{\prime} k^{\prime}}}{\left(s_{j^{\prime} k^{\prime}} \mathrm{R}_{2}\right.}, \\
& \lambda_{2}^{j k} \approx \Lambda \frac{\omega_{j^{2} k^{2}}}{\left(s_{j^{2} k^{2}}^{\mathrm{CN}}\right)^{2}} .
\end{aligned}
$$

In order to find the perturbative behavior of the first two Schmidt eigenvalues, one averages over the random variables $\omega_{j^{\prime} k^{\prime}}$ and $s_{j^{\prime} k^{\prime}} \mathrm{R}_{2}$ respectively $s_{j^{2} k^{2}}^{\mathrm{CN}}$. The distribution of $\omega_{j^{\prime} k^{\prime}}$ is given by Eq. 20. In order to perform the averaging for the first Schmidt eigenvalue define

$$
R_{j k}(s, \omega)=\sum_{j^{\prime} k^{\prime} \neq j k} \delta\left(\omega-\omega_{j^{\prime} k^{\prime}}\right) \delta\left(s-s_{j^{\prime} k^{\prime}}^{\mathrm{R}_{2}}\right)
$$

as probability density to find a level with distance $s_{j^{\prime} k^{\prime}}^{\mathrm{R}_{2}}$ to $\theta_{j k}$ and a corresponding matrix element $\omega$ with the value $\omega_{j k}$. Under the assumption that the matrix elements and the spacings are uncorrelated, the ensemble average gives

$$
\overline{R(s, \omega)}=\rho_{V_{12}}(\omega) R_{2}(s)=\rho_{V_{12}}(\omega) .
$$

In the last equality the result

$$
R_{2}(s)=\overline{\sum_{j^{\prime} k^{\prime} \neq j k} \delta\left(s-s_{j^{\prime} k^{\prime}}^{\mathrm{R}_{2}}\right)}=1
$$

for a Poisson distributed random variable with $-\infty \leq s \leq \infty$ is used. The distribution of the closer neighbor level spacings $s_{j^{2} k^{2}}^{\mathrm{CN}}$ is given by [73]

$$
\rho_{\mathrm{CN}}(s)=2 \exp (-2 s) .
$$

Thus the result for the averaged Schmidt eigenvalues is

$$
\begin{aligned}
& \overline{\lambda_{1}}=1-\Lambda \int_{-\infty}^{\infty} \mathrm{d} s \int_{0}^{\infty} \mathrm{d} \omega \frac{\omega}{s^{2}} \rho_{V_{12}}(\omega), \\
& \overline{\lambda_{2}}=\Lambda \int_{0}^{\infty} \mathrm{d} s \int_{0}^{\infty} \mathrm{d} \omega \frac{\omega}{s^{2}} \rho_{V_{12}}(\omega) \rho_{\mathrm{CN}}(s) .
\end{aligned}
$$



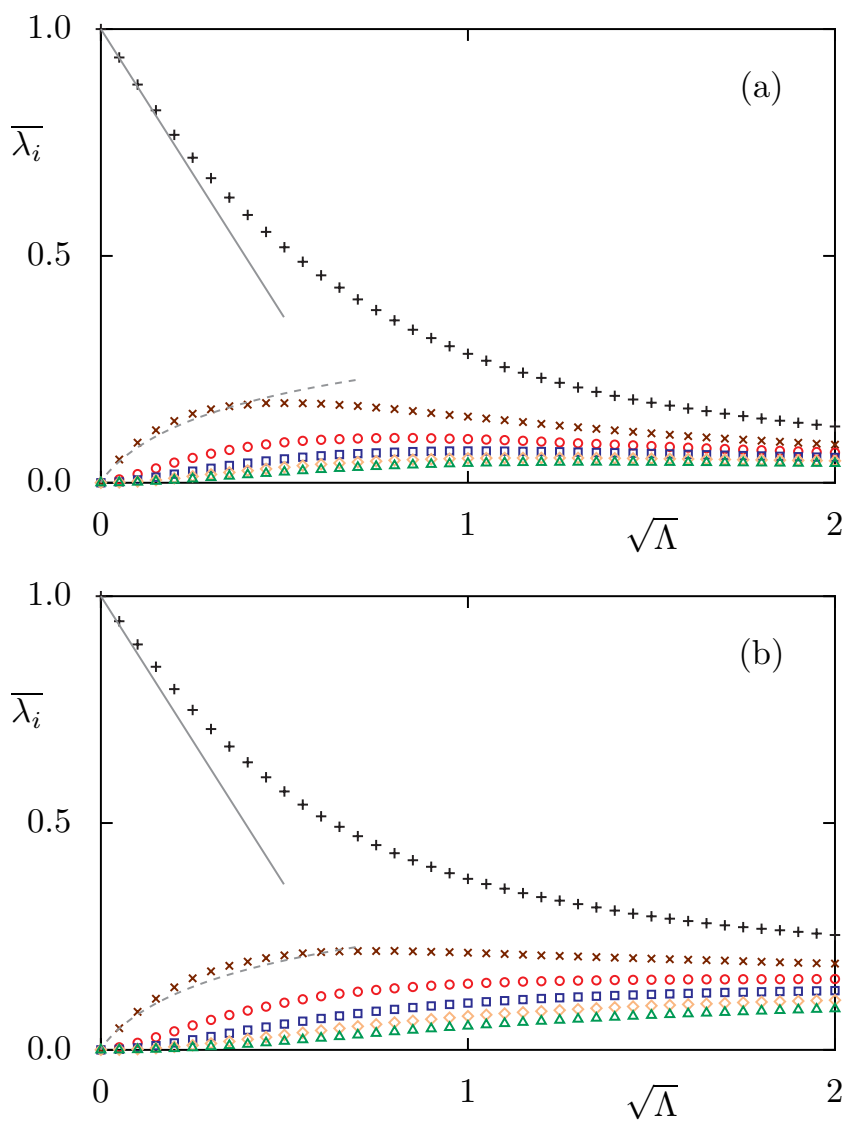

FIG. 4. Average Schmidt eigenvalues $\overline{\lambda_{i}}$ in dependence on $\sqrt{\Lambda}$ for $i=1,2, \ldots, 6$ (top to bottom with different symbols) for the coupled kicked tops for (a) equal dimensions $j_{1}=j_{2}=50$ and (b) different dimensions $\left(j_{1}, j_{2}\right)=(3,700)$. The solid grey line shows the prediction (58) for $\overline{\lambda_{1}}$, the dashed grey line shows the prediction (59). Parameters are $k_{1}=12.0, k_{2}=15.0, \alpha_{1}=0.35$, and $\alpha_{2}=0.4$.

Due to the singularity of the integrands for $s \rightarrow 0$, the integrals in Eq. (55) and Eq. (56) diverge. Following Ref. [34] we therefore perform the replacement

$$
\frac{\Lambda \omega}{s^{2}} \rightarrow \frac{1}{2}\left(1-\frac{|s|}{\sqrt{s^{2}+4 \Lambda \omega}}\right) .
$$

This regularization is the correct description for small $s$ and has the same asymptotics for large $s$. Therefore this replacement is used in the entire domain of integration.

For the coupling 27] for the coupled kicked tops the distribution $\rho_{V_{12}}(\omega)$ is that of the random matrix transition ensemble with product phases defined in Sec. II B 3 and therefore given by Eq. (20). Using this in Eqs. (55) and (56), together with Eq. 54, we obtain

$$
\begin{aligned}
\overline{\lambda_{1}} & =1-\int_{0}^{\infty} \mathrm{d} s \int_{0}^{\infty} \mathrm{d} \omega\left(1-\frac{s}{\sqrt{s^{2}+4 \Lambda \omega}}\right) \frac{\mathrm{K}_{0}(\sqrt{\omega})}{\pi \sqrt{\omega}} \\
& =1-\frac{4}{\pi} \sqrt{\Lambda}
\end{aligned}
$$

and

$$
\overline{\lambda_{2}}=\int_{0}^{\infty} \mathrm{d} s \int_{0}^{\infty} \mathrm{d} \omega\left(1-\frac{s}{\sqrt{s^{2}+4 \Lambda \omega}}\right) \mathrm{e}^{-2 s} \frac{\mathrm{K}_{0}(\sqrt{\omega})}{\pi \sqrt{\omega}} .
$$

A comparison of these predictions with the average Schmidt eigenvalues $\overline{\lambda_{i}}$, where the average is done over all eigenstates, of the coupled kicked tops is shown in Fig. 4 a). Here, we evaluate the integral in Eq. (59) numerically. Good agreement for small values of $\sqrt{\Lambda}$ is found.

Note that the predictions (58) and (59) are based on the specific coupling (17). If one uses the coupling (11) introduced for the random matrix transition ensemble in Ref. [32], see Sec. II B 2, one obtains for the COE case the results given in App. B, while the results for the CUE were obtained in Refs. [32, 34].

The above derivation equally applies to the case of different dimensionalities. Thus in Eqs. (58) and (59) only the correct transition parameter $\Lambda$, computed via Eq. (4) and Eqs. (A22)(A24), has to be used. Already starting from $j_{1}=3$ and large $j_{2}$ good agreement is found, see Fig. 4(b), though in comparison with Fig. 4 (a) the regime of agreement for $\overline{\lambda_{1}}$ is smaller.

\section{Entanglement entropies}

First we consider the perturbative description of the entanglement entropies $S_{\alpha}$ for small $\sqrt{\Lambda}$. To use Eq. (44) an expression for the moments $\mu_{\alpha}$ is required. For this we split the sum in the definition of $\mu_{\alpha}$ in Eq. (43) into two parts and consider $\overline{\lambda_{1}^{\alpha}}$ and $\sum_{j>1} \overline{\lambda_{j}^{\alpha}}$ separately. For $\overline{\lambda_{1}^{\alpha}}$ it is shown in Ref. [34] that the leading order result can be written as

$$
\begin{aligned}
\overline{\lambda_{1}^{\alpha}}= & +2 \int_{0}^{\infty} \mathrm{d} s \int_{0}^{\infty} \mathrm{d} \omega \rho_{V_{12}}(\omega) \\
& \times\left[\left(1-\frac{1}{2}\left(1-\frac{s}{\sqrt{s^{2}+4 \Lambda \omega}}\right)\right)^{\alpha}-1\right] \\
& +O(\Lambda),
\end{aligned}
$$

and the corrections of order $O(\Lambda)$ are given as

$$
\begin{aligned}
& 2 \int_{0}^{\infty} \mathrm{d} s_{1} \int_{0}^{\infty} \mathrm{d} \omega_{1} \int_{0}^{\infty} \mathrm{d} s_{2} \int_{0}^{\infty} \mathrm{d} \omega_{2} \rho_{V_{12}}\left(\omega_{1}\right) \rho_{V_{12}}\left(\omega_{2}\right) \\
& \times\left[1+\left(1-\frac{f\left(s_{1}, \omega_{1}\right)}{2}-\frac{f\left(s_{2}, \omega_{2}\right)}{2}\right)^{\alpha}\right. \\
& \left.\quad-\left(1-\frac{f\left(s_{1}, \omega_{1}\right)}{2}\right)^{\alpha}-\left(1-\frac{f\left(s_{2}, \omega_{2}\right)}{2}\right)^{\alpha}\right]
\end{aligned}
$$

Here the abbreviation $f(s, \omega)=1-s /\left(\sqrt{s^{2}+4 \Lambda \omega}\right)$ is used. Using the density $\rho_{V_{12}}(\omega)$ from Eq. 20 for the coupled kicked tops this leads to

$$
\overline{\lambda_{1}^{\alpha}}=1-C_{1}(\alpha) \sqrt{\Lambda}+C_{3}(\alpha) \Lambda
$$


with

$$
\begin{aligned}
C_{1}(\alpha)= & \frac{2}{\pi} \int_{0}^{\frac{1}{2}} \mathrm{~d} t \frac{1-(1-t)^{\alpha}}{t^{3 / 2}(1-t)^{3 / 2}} \\
= & \frac{4 \sqrt{2}}{\pi}{ }_{2} F_{1}\left(-\frac{1}{2}, \frac{3}{2}-\alpha ; \frac{1}{2} ; \frac{1}{2}\right), \\
C_{3}(\alpha)= & \frac{2}{\pi^{2}} \int_{0}^{\frac{1}{2}} \mathrm{~d} t_{1} \int_{0}^{\frac{1}{2}} \mathrm{~d} t_{2} \\
& \times \frac{1+\left(1-t_{1}-t_{2}\right)^{\alpha}-\left(1-t_{1}\right)^{\alpha}-\left(1-t_{2}\right)^{\alpha}}{t_{1}^{3 / 2}\left(1-t_{1}\right)^{3 / 2} t_{2}^{3 / 2}\left(1-t_{2}\right)^{3 / 2}} .
\end{aligned}
$$

Here ${ }_{2} F_{1}$ is Gauss' hypergeometric function [66, Eq. 15.2.1]. For $\alpha=1$ Eq. (62) reproduces the prediction Eq. (58) for $\overline{\lambda_{1}}$. To calculate $\sum_{j>1} \overline{\lambda_{j}^{\alpha}}$ we use [34]

$$
\sum_{j>1} \overline{\lambda_{j}^{\alpha}}=\int_{-\infty}^{\infty} \mathrm{d} s \int_{0}^{\infty} \mathrm{d} \omega \frac{\rho_{V_{12}}(\omega)}{2^{\alpha}}\left(1-\frac{|s|}{\sqrt{s^{2}+4 \Lambda \omega}}\right)^{\alpha} .
$$

Inserting Eq. 20) for $\rho_{V_{12}}(\omega)$ leads to

$$
\sum_{j>1} \overline{\lambda_{j}^{\alpha}}=C_{2}(\alpha) \sqrt{\Lambda}
$$

with

$$
\begin{aligned}
C_{2}(\alpha) & =\frac{2}{\pi} \int_{0}^{\frac{1}{2}} \frac{t^{\alpha}}{t^{3 / 2}(1-t)^{3 / 2}} \\
& =\frac{2}{\pi} B_{1 / 2}\left(\alpha-\frac{1}{2},-\frac{1}{2}\right) .
\end{aligned}
$$

Here $B_{z}(a, b)$ is the incomplete Beta function [66, Eq. 8.17.1]. With this it is now possible to write the average moments $\overline{\mu_{\alpha}}$ as

$$
\overline{\mu_{\alpha}}=1-C(\alpha) \sqrt{\Lambda}+C_{3}(\alpha) \Lambda
$$

where

$$
C(\alpha)=C_{1}(\alpha)-C_{2}(\alpha)=\frac{4}{\sqrt{\pi}} \frac{\Gamma\left(\alpha-\frac{1}{2}\right)}{\Gamma(\alpha-1)} .
$$

This results in

$$
\overline{S_{\alpha}}=\frac{4}{\sqrt{\pi}} \frac{\Gamma\left(\alpha-\frac{1}{2}\right)}{\Gamma(\alpha)} \sqrt{\Lambda}-\frac{C_{3}(\alpha)}{\alpha-1} \Lambda
$$

as an approximation of the entropies for small $\Lambda$. An important special case is the von Neumann entropy obtained in the limit $\alpha \rightarrow 1$, which gives

$$
\overline{S_{1}}=4 \sqrt{\Lambda}-\left(\frac{4}{\pi}-1\right) \Lambda .
$$

In addition to this perturbative description of $\overline{S_{\alpha}}$, valid for small $\sqrt{\Lambda}$, the recursively embedded perturbation theory can be applied following Ref. [34] to obtain a complete description of the entropies as a function of $\Lambda$. The underlying idea

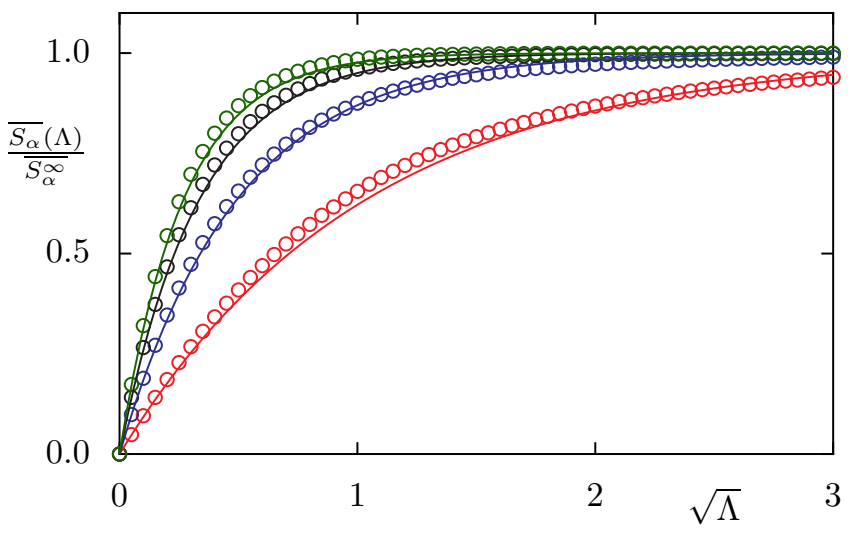

FIG. 5. Average entanglement entropies $\overline{S_{\alpha}}$, rescaled by $\overline{S_{\alpha}^{\infty}}$, in dependence on $\sqrt{\Lambda}$ for $\alpha=1,2,3,4$ (bottom to top) for the coupled kicked tops. The solid curves show the prediction as given by Eq. (74) and Eq. 75. Parameters are $j_{1}=j_{2}=50, k_{1}=12.0$, $k_{2}=15.0, \alpha_{1}=0.35$, and $\alpha_{2}=0.4$.

is that with increasing $\Lambda$ successively more and more Schmidt eigenvalues become relevant. This can be accounted for by a recursive description which can be approximated by a differential equation. Furthermore, the maximal values of the entropies for the fully entangled situation are used, which follow from the moments of the Marčenko-Pastur distribution of the Schmidt eigenvalues [18]. Restricting to $N=N_{1}=N_{2}$ one has

$$
\begin{aligned}
& \overline{S_{1}^{\infty}}=\ln N-\frac{1}{2}, \\
& \overline{S_{\alpha}^{\infty}}=\frac{1-\mathcal{C}_{\alpha} N^{1-\alpha}}{\alpha-1} \quad \text { for } \alpha>1,
\end{aligned}
$$

where $\mathcal{C}_{\alpha}=\frac{1}{\alpha+1}\left(\begin{array}{c}2 \alpha \\ \alpha\end{array}\right)$ are Catalan numbers [66, §26.5.]. This leads to

$$
\overline{S_{\alpha}(\Lambda)} \approx\left[1-\exp \left(-\frac{C(\alpha)}{(\alpha-1) \overline{S_{\alpha}^{\infty}}} \sqrt{\Lambda}\right)\right] \overline{S_{\alpha}^{\infty}}
$$

as prediction for the entropies. In particular, using $\lim _{\alpha \rightarrow 1} \frac{C(\alpha)}{\alpha-1}=4$ gives for the von Neumann entropy,

$$
\overline{S_{1}(\Lambda)} \approx\left[1-\exp \left(-\frac{4}{\overline{S_{1}^{\infty}}} \sqrt{\Lambda}\right)\right] \overline{S_{1}^{\infty}} .
$$

Figure 5 shows a comparison of the recursively embedded perturbation theory predictions with the results for the coupled kicked tops. The agreement between the curves is overall very good.

\section{Entanglement entropies for different dimensions}

To describe the entanglement entropies for different subsystem dimensions the recursively embedded perturbation theory can be applied as well. We restrict to the case of the linear 


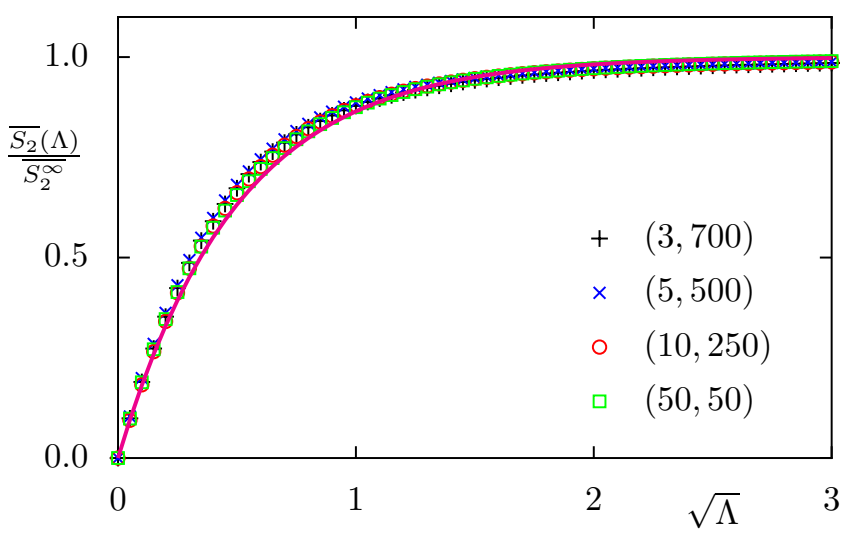

FIG. 6. Rescaled linear entropy, $\overline{S_{2}} / \overline{S_{2}^{\infty}}$, in dependence on $\sqrt{\Lambda}$ for different dimensions $\left(j_{1}, j_{2}\right)=$ $(3,700),(5,500),(10,250),(50,50)$ of the coupled kicked tops. The solid magenta curve shows Eq. 76 using $\overline{S_{2}^{\infty}}=1$ which corresponds to $N_{1}, N_{2} \rightarrow \infty$. Parameters are $k_{1}=12.0$, $k_{2}=15.0, \alpha_{1}=0.35$, and $\alpha_{2}=0.4$.

entropy obtained for $\alpha=2$. For this the maximum of the entropy $\overline{S_{2}^{\infty}}$ is exactly given by Lubkin's result 47 . This leads to the prediction for the linear entropy

$$
\overline{S_{2}(\Lambda)} \approx\left[1-\exp \left(-\frac{2}{\overline{S_{2}^{\infty}}} \sqrt{\Lambda}\right)\right] \overline{S_{2}^{\infty}}
$$

as by Eq. 69) one has $C(2)=2$. Thus while the functional dependence is the same as in Eq. (74), the different dimensionalities of the subsystems are accounted for by the formula for $\overline{S_{2}^{\infty}}$ and the dependence of the transition parameter $\Lambda$ on the subsystem dimensions. Note that for the other entropies with $\alpha \neq 2$, the maximal values of the entropies corresponding to Eqs. (72) and (73) follow from the results in Ref. [18].

Figure 6 shows the rescaled linear entropy for the coupled kicked tops for several pairs of different dimensions as well as the prediction from Eq. (76). From this plot it can be seen that for $j_{1} \geq 3$ the linear entropy of the coupled kicked tops for different dimensions, after rescaling by the corresponding $\overline{S_{2}^{\infty}}$ given by Eq. 47, collapse rather well to one universal curve described by Eq. (76).

Thus we get a remarkable range of universal behavior and agreement with the theory $(76)$. Only for the very small system sizes $j_{1}=1,2$ (not shown) there are systematic differences and a detailed understanding and theoretical description in this case is an interesting open question for the future.

\section{E. Statistics of Schmidt eigenvalues}

The average Schmidt eigenvalues and the average entanglement entropies provide a compact characterization of the possible amount of entanglement in dependence of the universal scaling parameter $\Lambda$. More detailed information is obtained by considering the statistics of the whole spectrum of Schmidt eigenvalues [24], or the entanglement spectrum [74]. For large $\Lambda$ one expects that the distribution of the scaled
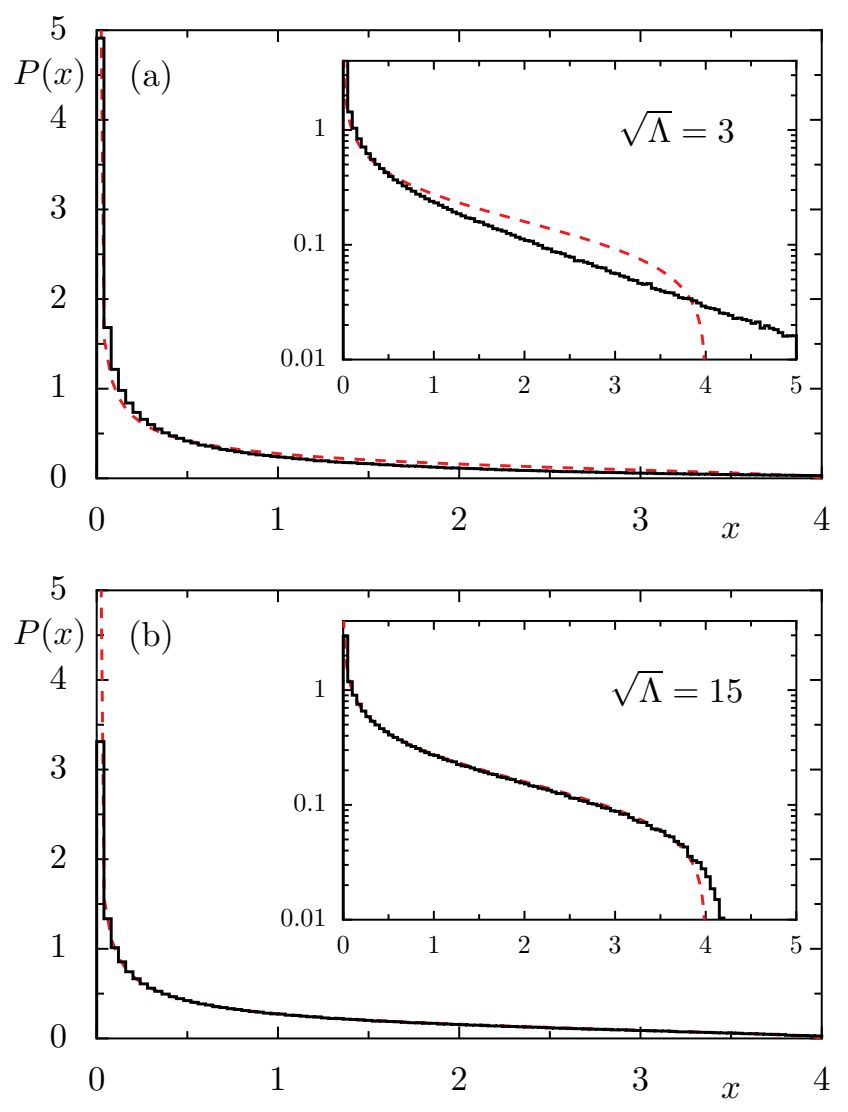

FIG. 7. Distribution $P(x)$ of the re-scaled Schmidt eigenvalues $x_{i}=$ $\lambda_{i} N_{1}$ for (a) $\sqrt{\Lambda}=3$ and (b) $\sqrt{\Lambda}=15$, both with $N_{1}=2 j_{1}+1$ for $j_{1}=j_{2}=50$. The red dashed curve shows the Marčenko-Pastur distribution (78) for $Q=1$. The insets show the same data in a semi-logarithmic plot.

Schmidt eigenvalues

$$
x_{i}=\lambda_{i} N_{1}
$$

is given by the Marčenko-Pastur distribution, when $N_{1}$ and $N_{2}$ are large but their ratio $Q=N_{2} / N_{1} \geq 1$ is fixed [18]. This distribution reads [17]

$$
P_{\mathrm{MP}}^{Q}(x)=\frac{Q}{2 \pi} \frac{\sqrt{\left(x_{+}-x\right)\left(x-x_{-}\right)}}{x}, x_{-} \leq x \leq x_{+},
$$

where

$$
x_{ \pm}=1+\frac{1}{Q} \pm \frac{2}{\sqrt{Q}}
$$

For chaotic states of coupled kicked tops, i.e. in the regime of large $\Lambda$, this has been verified in Ref. [24]. Exact results for finite $N_{1}$ were obtained in Refs. [45, 46].

We want to investigate the dependence of the distribution of the scaled Schmidt eigenvalues on $\Lambda$ for equal subsystem dimensions as well as for different dimensions. In the uncoupled case, i.e. at $\Lambda=0$, all eigenstates are unentangled such that $\lambda_{1}=1$ and $\lambda_{i}=0$ for $i>1$ leading to 

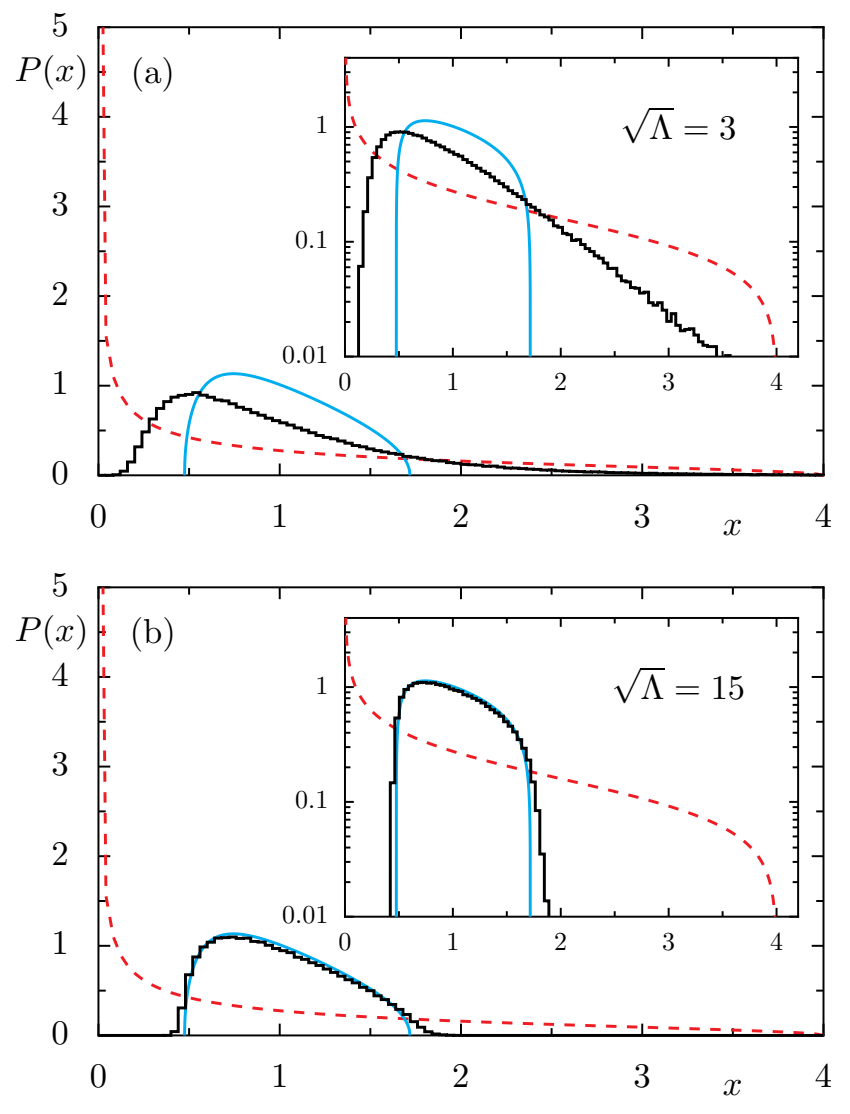

FIG. 8. Distribution $P(x)$ of the re-scaled Schmidt eigenvalues $x_{i}=$ $\lambda_{i} N_{1}$ for (a) $\sqrt{\Lambda}=3$ and (b) $\sqrt{\Lambda}=15$, both with $N_{1}=2 j_{1}+1$ for $\left(j_{1}, j_{2}\right)=(15,160)$. The full cyan curve shows the MarčenkoPastur distribution 78 for $Q=\left(2 j_{1}+1\right) /\left(2 j_{2}+1\right)=0.0966$ and the red dashed curve for $Q=1$. The insets show the same data in a semi-logarithmic plot.

$P(x)=\left(1-1 / N_{1}\right) \delta(x)+1 / N_{1} \delta\left(x-N_{1}\right)$. With increasing $\sqrt{\Lambda}$ the distribution $P(x)$ of the re-scaled Schmidt eigenvalues (77) will move towards the Marčenko-Pastur distribution. Figure 7 shows the result for the case of equal dimension of the subsystems and illustrates that this transition is rather slow as even for $\sqrt{\Lambda}=15$ small deviations are visible near $x=4$. These deviations are due to the finite system size and become smaller with increasing $j_{1}=j_{2}$. The slow convergence with $\sqrt{\Lambda}$ to the Marčenko-Pastur distribution has already been observed for the example of the coupled standard maps in Ref. [34], for which, however, the transition appears to be slightly faster, which is consistent with the observations for the spectral statistics made in Sec. III A.

The case of different dimensions of the subsystems is shown in Fig. 8. Again for rather large $\sqrt{\Lambda}$ good agreement between the distribution for the coupled kicked tops and the Marčenko-Pastur distribution is found. Interestingly, the distribution is quite concentrated around $x=1$, so that one could think that there are some states which are close to maximal entanglement, i.e. $\lambda_{i}=1 / N_{1}$ for all $i$. However, we observe, that this is not the case at least for a finite $N_{2}$. To obtain maximal entanglement, sophisticated protocols are needed [75].

\section{SUMMARY AND OUTLOOK}

For bipartite systems the spectral statistics and entanglement of eigenstates are investigated in dependence of a tunable interaction. We focus on classically fully chaotic subsystem which can be modeled by circular unitary or orthogonal ensembles and derive an exact expression for the ensemble average of the transition parameter. By specifying the statistical properties of the coupling between the subsystems, different random matrix transition ensembles are obtained. In particular assuming a product structure for the coupling allows for explicitly describing the dependence of the transition parameter on the individual subsystems Hilbert space dimensions and the coupling strength. An important model system following COE statistics is given by two coupled kicked tops. We utilize this system in order to illustrate the transition from non-interacting to random matrix behavior. To this end we consider the level spacing distribution in the case of equal and unequal dimensions of the subsystems. For equal dimensions the statistics depends solely on the transition parameter. For unequal dimensions we find deviations if one of the subsystems has a small dimension. However, universality is already achieved when the smaller subsystem has dimension larger than ten. For large transition parameter the limiting case of Wigner distributed level spacings is approached slower than for systems described by the CUE transition ensemble. A perturbative description, obtained from the random matrix transition ensemble with product phases, is in good agreement with numerical results.

For the average entanglement of eigenstates, in terms of their HCT entropies including the von Neumann entropy, a universal scaling for both equal and unequal dimensions is found. Only if the dimension of one subsystem is smaller than five, deviations from universality are observed. Applying perturbation theory for the average first and second Schmidt eigenvalues gives very good agreement with the numerical results for the coupled kicked tops for equal and unequal dimensions for small transition parameters. Using the recursively embedded perturbation theory allows to extend the perturbative description towards the large coupling regime. Very good agreement of the HCT entropies and the von Neumann entropy with the numerical results is found for all values of the transition parameter. Finally we study the distribution of Schmidt eigenvalues for which a rather slow transition from the unentangled case towards Marčenko-Pastur distribution for both equal and unequal dimensions is observed.

The results presented in this paper confirm that the theory based on the transition parameter gives rise to an accurate description of eigenstate entanglement for bipartite systems satisfying a unitary symmetry when the specific structure of the coupling is taken into account. There are several interesting open questions. The observed deviations from universality if one subsystem is very small are not well captured by the asymptotic results of the random matrix transition ensemble, but potentially are accessible by analytic approaches. Thus studying those small systems may give rise to further insight both in terms of eigenstate entanglement as well as in the time evolution of initially pure states. Another interesting question 
for the future is to find an analytical expression for the transition between the distribution of the Schmidt eigenvalues at $\Lambda=0$ and the Marčenko-Pastur distribution based on the random matrix transition ensemble. Furthermore, as the system of two coupled kicked tops may be interpreted as the collective dynamics of two spin chains with non-local interaction one may ask to what extent the results transfer to interacting many-body systems.

\section{ACKNOWLEDGMENTS}

We thank Roland Ketzmerick, Arul Lakshminarayan, Jan Schmidt, Shashi Srivastava, and Steve Tomsovic for useful discussions.

\section{Appendix A: Computation of the transition parameter}

\section{1. $\mathrm{COE}$}

If the bipartite system is such that the individual subsystems are described by random matrix theory in the presence of an anti-unitary symmetry, one can set up the random matrix transition ensemble (8) as

$$
U_{\mathrm{COE}}(\varepsilon)=U_{12}(\varepsilon)\left(U_{1}^{\mathrm{COE}} \otimes U_{2}^{\mathrm{COE}}\right),
$$

where $U_{1}^{\mathrm{COE}}$ und $U_{2}^{\mathrm{COE}}$ are independently chosen COE random matrices of dimension $N_{1} \times N_{1}$ and $N_{2} \times N_{2}$, respectively. The interaction $U_{12} \equiv U_{12}(\varepsilon)$ is a diagonal unitary matrix of dimension $N_{1} N_{2} \times N_{1} N_{2}$. For the moment we do not yet specify the statistics of its entries.

We now determine the transition parameter $\Lambda=v^{2} / D^{2}$, where $v^{2}$ is the mean square of the off-diagonal elements for $U_{12}$ in the basis in which $U_{1}^{\mathrm{COE}} \otimes U_{2}^{\mathrm{COE}}$ is diagonal and $D$ is the mean level spacing. For this consider $\Omega_{i}=E_{i}^{\dagger} U_{i}^{\mathrm{COE}} E_{i}$, where $E_{i}$ is the matrix containing the eigenvectors of $U_{i}^{\mathrm{COE}}$ as columns. Defining $\Upsilon=E_{1} \otimes E_{2}$ we get the representation of $U_{12}$ in the requested basis, $\Gamma=\Upsilon^{\dagger} U_{12} \Upsilon$, where

$$
\gamma_{i l}=(\Gamma)_{i l}=\sum_{j, k=1}^{N_{1} N_{2}} v_{k i}^{*}\left(U_{12}\right)_{k j} v_{j l}
$$

with $v_{j l}=(\Upsilon)_{j l}$.Thus

$$
\begin{aligned}
v^{2} & =\frac{\sum_{i, l=1}^{N_{1} N_{2}}\left|\gamma_{i l}\right|^{2}-\sum_{i=1}^{N_{1} N_{2}}\left|\gamma_{i i}\right|^{2}}{\left(N_{1} N_{2}\right)^{2}-N_{1} N_{2}} \\
& =\frac{N_{1} N_{2}-\sum_{i=1}^{N_{1} N_{2}}\left|\gamma_{i i}\right|^{2}}{N_{1} N_{2}\left(N_{1} N_{2}-1\right)},
\end{aligned}
$$

where in the second equality the unitarity of $\Gamma$ has been used. Next the sum over the diagonal elements is determined

$$
\begin{aligned}
\sum_{i=1}^{N_{1} N_{2}}\left|\gamma_{i i}\right|^{2} & =\sum_{i=1}^{N_{1} N_{2}} \sum_{k=1}^{N_{1} N_{2}} v_{k i}^{*}\left(U_{12}\right)_{k k} v_{k i} \sum_{l=1}^{N_{1} N_{2}} v_{l i}\left(U_{12}^{*}\right)_{l l} v_{l i}^{*} \\
& =\sum_{k, l=1}^{N_{1} N_{2}}\left(U_{12}\right)_{k k}\left(U_{12}^{*}\right)_{l l} \sum_{i=1}^{N_{1} N_{2}}\left|v_{i k}\right|^{2}\left|v_{i l}\right|^{2} .
\end{aligned}
$$

Next the product $\left|v_{i k}\right|^{2}\left|v_{i l}\right|^{2}$ is replaced by its average $\overline{\left|v_{i k}\right|^{2}\left|v_{i l}\right|^{2}}$ over the COE. By the definition of $\Upsilon$ and the independence of $U_{1}^{\mathrm{COE}}$ and $U_{2}^{\mathrm{COE}}$ one gets

$$
\overline{\left|v_{i k}\right|^{2}\left|v_{i l}\right|^{2}}=\overline{\left|\left(E_{1}\right)_{i_{1} k_{1}}\right|^{2}\left|\left(E_{1}\right)_{i_{1} l_{1}}\right|^{2}} \overline{\left|\left(E_{2}\right)_{i_{2} k_{2}}\right|^{2}\left|\left(E_{2}\right)_{i_{2} l_{2}}\right|^{2}},
$$

where, due to the product structure, we identify $i \equiv\left(i_{1}, i_{2}\right)$, $k \equiv\left(k_{1}, k_{2}\right)$, and $l \equiv\left(l_{1}, l_{2}\right)$. For each term one has in case of the COE [76]

$$
\overline{\left|\left(E_{1}\right)_{i_{1} k_{1}}\right|^{2}\left|\left(E_{1}\right)_{i_{1} l_{1}}\right|^{2}}=\frac{2 \delta_{k_{1} l_{1}}+1}{N_{1}\left(N_{1}+2\right)} .
$$

Thus we get for the average in Eq. A5

$$
\begin{aligned}
\overline{\left|v_{i k}\right|^{2}\left|v_{i l}\right|^{2}} & =\frac{2 \delta_{k_{1} l_{1}}+1}{N_{1}\left(N_{1}+2\right)} \frac{2 \delta_{k_{2} l_{2}}+1}{N_{2}\left(N_{2}+2\right)} \\
& =\frac{4 \delta_{k_{1} l_{1}} \delta_{k_{2} l_{2}}+2 \delta_{k_{1} l_{1}}+2 \delta_{k_{2} l_{2}}+1}{N_{1}\left(N_{1}+2\right) N_{2}\left(N_{2}+2\right)} .
\end{aligned}
$$

As the right-hand side is independent of $i$, one gets

$$
\begin{aligned}
\sum_{i=1}^{N_{1} N_{2}}\left|\gamma_{i i}\right|^{2}= & \sum_{k_{1}, l_{1}=1}^{N_{1}} \sum_{k_{2}, l_{2}=1}^{N_{2}} \\
& \left(4 \delta_{k_{1} l_{1}} \delta_{k_{2} l_{2}}+2 \delta_{k_{1} l_{1}}+2 \delta_{k_{2} l_{2}}+1\right) \\
& \times \frac{\left\langle k_{1} k_{2}\left|U_{12}\right| k_{1} k_{2}\right\rangle\left\langle l_{1} l_{2}\left|U_{12}^{*}\right| l_{1} l_{2}\right\rangle}{\left(N_{1}+2\right)\left(N_{2}+2\right)}
\end{aligned}
$$

Using the partial traces and the Hilbert-Schmidt norm we arrive at

$$
\sum_{i=1}^{N_{1} N_{2}}\left|\gamma_{i i}\right|^{2}=\frac{4 N_{1} N_{2}+2\left\|U_{12}^{(1)}\right\|^{2}+2 \| U_{12}^{(2)}||^{2}+\left|\operatorname{tr}\left(U_{12}\right)\right|^{2}}{\left(N_{1}+2\right)\left(N_{2}+2\right)} .
$$

Insertion in Eq. A3 gives the final result (4) for the transition parameter in the COE case.

\section{CUE}

The derivation for the CUE follows the same steps as for the COE. The only difference is to replace relation A6 by the CUE result [77, Eq. (10)],

$$
\overline{\left|\left(E_{1}\right)_{i_{1} k_{1}}\right|^{2}\left|\left(E_{1}\right)_{i_{1} l_{1}}\right|^{2}}=\frac{\delta_{k_{1} l_{1}}+1}{N_{1}\left(N_{1}+1\right)} .
$$

With this the result $(6)$ is obtained.

\section{Random matrix transition ensemble}

For the random matrix transition ensemble introduced in Ref. [32], see Sec. II B 2, the interaction is given by the diagonal matrix with random phases. In this case the partial traces and Hilbert-Schmidt norms have been derived in 
Refs. [32, 34] as

$$
\begin{aligned}
& \overline{\left|\operatorname{tr}\left(U_{12}^{\mathrm{RMT}}\right)\right|^{2}}=N_{1} N_{2}\left(1+\left(N_{1} N_{2}-1\right) \frac{\sin ^{2}(\pi \varepsilon)}{\pi^{2} \varepsilon^{2}}\right), \\
& \overline{\left\|U_{12}^{\mathrm{RMT}^{(1)}}\right\|^{2}}=N_{1} N_{2}\left(1+\left(N_{2}-1\right) \frac{\sin ^{2}(\pi \varepsilon)}{\pi^{2} \varepsilon^{2}}\right), \\
& \overline{\left\|U_{12}^{\mathrm{RMT}^{(2)}}\right\|^{2}}=N_{1} N_{2}\left(1+\left(N_{1}-1\right) \frac{\sin ^{2}(\pi \varepsilon)}{\pi^{2} \varepsilon^{2}}\right) .
\end{aligned}
$$

Using these in Eq. (4) and Eq. (6), respectively, gives the corresponding results Eq. (12) for the COE and Eq. (13) for the CUE.

\section{Random matrix transition ensemble with product phases}

To calculate the transition parameter for the random matrix transition ensemble with product phases, see Sec. II B 3, we first consider the squared norm of the trace and the HilbertSchmidt norm of the partial traces of the interaction $U_{12}(\varepsilon)$ with the coupling in Eq. (17). To this end we collect the random variables into the vectors $\xi=\left(\xi_{j}\right)_{j=1, \ldots, N_{1}}$ and $\tilde{\xi}=\left(\tilde{\xi}_{k}\right)_{k=1, \ldots, N_{2}}$ where all components are i.i.d. distributed uniformly on $[-1 / 2,1 / 2]$. Averaging over the random variables in the coupling allows for writing

$$
\overline{\left|\operatorname{tr}\left(U_{12}\right)\right|^{2}}=\sum_{j, j^{\prime}=1}^{N_{1}} \sum_{k, k^{\prime}=1}^{N_{2}} \int_{[-1 / 2,1 / 2]^{N_{1}+N_{2}}} \mathrm{~d} \xi \mathrm{d} \tilde{\xi} \mathrm{e}^{2 \pi \mathrm{i} \varepsilon\left(\xi_{j} \tilde{\xi}_{k}-\xi_{j^{\prime}} \tilde{\xi}_{k^{\prime}}\right)} .
$$

For each term in this fourfold sum the integrand depends at most on four of the integration variables, namely if $j \neq j^{\prime}$ and $k \neq k^{\prime}$, and we can integrate over the remaining random variables each giving a factor of one. This gives

$$
\int_{[-1 / 2,1 / 2]^{4}} \mathrm{~d} \xi_{j} \mathrm{~d} \xi_{j^{\prime}} \mathrm{d} \tilde{\xi}_{k} \mathrm{~d} \tilde{\xi}_{k^{\prime}} \mathrm{e}^{2 \pi \mathrm{i} \varepsilon\left(\xi_{j} \tilde{\xi}_{k}-\xi_{j^{\prime}} \tilde{\xi}_{k^{\prime}}\right)}=\frac{4}{\varepsilon^{2} \pi^{2}} \mathrm{Si}\left(\frac{\varepsilon \pi}{2}\right)^{2},
$$

where $\operatorname{Si}(x)$ is the sine integral [66, Eq. 6.2.9]. Moreover it does not depend on the values of $j, j^{\prime}, k$ and $k^{\prime}$ and there are $N_{1} N_{2}\left(N_{1} N_{2}-N_{1} N_{2}+1\right)$ possible combinations of indices for this case. Furthermore, there are $N_{1} N_{2}\left(N_{2}-1\right)$ cases for which $j=j^{\prime}$ and $k \neq k^{\prime}$ and $N_{2} N_{1}\left(N_{1}-1\right)$ cases for which $j \neq j^{\prime}$ and $k=k^{\prime}$ and for which the integrand in Eq. A14) depends on three integration variables only. Finally, there are $N_{1} N_{2}$ cases for which $j=j^{\prime}$ and $k=k^{\prime}$ where the integrand depends on two integration variables only. In all cases the corresponding integrals can be evaluated analytically. Combining the integrals and taking the frequency of their appearance into account gives

$$
\begin{aligned}
\overline{\left|\operatorname{tr}\left(U_{12}\right)\right|^{2}}= & N_{1} N_{2}\left(1+\frac{2}{\varepsilon^{2} \pi^{2}}\left[\left(N_{1}+N_{2}-2\right) \chi(\varepsilon \pi)\right.\right. \\
& \left.\left.+2\left(N_{1}-1\right)\left(N_{2}-1\right) \operatorname{Si}\left(\frac{\varepsilon \pi}{2}\right)^{2}\right]\right), \quad
\end{aligned}
$$

where $\chi(x)=x \operatorname{Si}(x)+\cos (x)-1$ has been used as abbreviation. Using the same arguments one finds

$$
\begin{aligned}
& \overline{\left\|U_{12}^{(1)}\right\|^{2}}=N_{1} N_{2}\left(1+\left(N_{2}-1\right) \frac{2 \chi(\varepsilon \pi)}{\varepsilon^{2} \pi^{2}}\right), \\
& \overline{\left\|U_{12}^{(2)}\right\|^{2}}=N_{1} N_{2}\left(1+\left(N_{1}-1\right) \frac{2 \chi(\varepsilon \pi)}{\varepsilon^{2} \pi^{2}}\right) .
\end{aligned}
$$

The transition parameter for the COE follows by inserting Eqs. A16, (A17), and (A18) in Eq. (4), and for the CUE via Eq. (6).

\section{Coupled kicked tops}

To determine the transition parameter for the coupled kicked tops, the partial traces and Hilbert-Schmidt norm have to be computed for the interaction $U_{12}(\varepsilon)$ as defined in Eq. 27]. The result is

$$
\begin{aligned}
\left|\operatorname{tr}\left(U_{12}\right)\right|^{2} & =\sum_{m_{1}, s_{1}=-j_{1}}^{j_{1}} \sum_{m_{2}, s_{2}=-j_{2}}^{j_{2}} \mathcal{E}\left(s_{1}, s_{2}\right) \mathcal{E}\left(m_{1},-m_{2}\right) \\
\left\|U_{12}^{(1)}\right\|^{2} & =\sum_{s_{1}=-j_{1}}^{j_{1}} \sum_{s_{2}, m_{2}=-j_{2}}^{j_{2}} \mathcal{E}\left(s_{1}, s_{2}\right) \mathcal{E}\left(s_{1},-m_{2}\right), \quad \text { (A20) } \\
\left\|U_{12}^{(2)}\right\|^{2} & =\sum_{s_{1}, m_{1}=-j_{1}}^{j_{1}} \sum_{s_{2}=-j_{2}}^{j_{2}} \mathcal{E}\left(s_{1}, s_{2}\right) \mathcal{E}\left(m_{1},-s_{2}\right)
\end{aligned}
$$

where $\mathcal{E}(s, m)=\exp \left(-\mathrm{i} \frac{\varepsilon}{\sqrt{j_{1} j_{2}}} s m\right)$ has been used as abbreviation.

For large $j_{1}, j_{2}$ the sums can be approximated by integrals which can be evaluated exactly, giving

$$
\begin{aligned}
\left|\operatorname{tr}\left(U_{12}\right)\right|^{2} & \approx\left(4 \frac{\sqrt{j_{1} j_{2}}}{\varepsilon} \operatorname{Si}(\varkappa / 2)\right)^{2}, \\
\left\|U_{12}^{(1)}\right\|^{2} & \approx 8 \frac{j_{1} j_{2}}{\varepsilon^{2} N_{1}} \chi(\varkappa), \\
\left\|U_{12}^{(2)}\right\|^{2} & \approx 8 \frac{j_{1} j_{2}}{\varepsilon^{2} N_{2}} \chi(\varkappa),
\end{aligned}
$$

where $\varkappa:=\frac{\varepsilon N_{1} N_{2}}{2 \sqrt{j_{1} j_{2}}}$. The explicit expression of the transition parameter $\Lambda$ is then obtained using Eq. (4).

For very large $\varepsilon$ the transition parameter $\Lambda$, determined from Eq. (4) and Eqs. (A22)- A24) saturates with value

$$
\Lambda_{\max }=\frac{N_{1}^{2} N_{2}^{2}\left(N_{1} N_{2}+2\left(N_{1}+N_{2}\right)\right)}{4 \pi^{2}\left(N_{1} N_{2}-1\right)\left(N_{1}+2\right)\left(N_{2}+2\right)} .
$$

Thus for fixed $N_{1}$ and $N_{2}$ it is not possible to obtain arbitrarily large $\Lambda$.

Note that for the interaction (27) of the coupled kicked tops choosing the specific value $\varepsilon=2 \pi$ gives $U_{12}(\varepsilon)=\mathrm{Id}$. This operator does not create any interaction between the two 
kicked tops. This illustrates the limits of the applicability of the transition parameter which has been obtained from perturbation theory and therefore provides the correct description for small values of $\varepsilon$ only.

\section{Appendix B: COE random matrix transition ensemble}

In this appendix for completeness we derive results for the entropies for the COE random matrix transition ensemble defined in Eq. 111. The results for the CUE case have been obtained in Ref. [33, 34].

The perturbative behavior of $\lambda_{1}$ and $\lambda_{2}$ can be determined following the steps in Sec. IVB using the coupling (15). The result is

$$
\begin{aligned}
\overline{\lambda_{1}} & =1-\int_{0}^{\infty} \mathrm{d} s \int_{0}^{\infty} \mathrm{d} \omega\left(1-\frac{s}{\sqrt{s^{2}+4 \Lambda \omega}}\right) \frac{\mathrm{e}^{-\omega / 2}}{\sqrt{2 \pi \omega}} \\
& =1-\frac{4}{\sqrt{2 \pi}} \sqrt{\Lambda}
\end{aligned}
$$

and

$$
\overline{\lambda_{2}}=\int_{0}^{\infty} \mathrm{d} s \int_{0}^{\infty} \mathrm{d} \omega\left(1-\frac{s}{\sqrt{s^{2}+4 \Lambda \omega}}\right) \mathrm{e}^{-2 s} \frac{\mathrm{e}^{-\omega / 2}}{\sqrt{2 \pi \omega}} .
$$

The perturbative description of the entanglement entropies $S_{\alpha}$ is given by the averaged moments $\overline{\mu_{\alpha}}$ in Eq. (68) with the terms

$$
C(\alpha)=C_{1}(\alpha)-C_{2}(\alpha)=2 \sqrt{2} \frac{\Gamma\left(\alpha-\frac{1}{2}\right)}{\Gamma(\alpha-1)},
$$

using

$$
\begin{aligned}
C_{1}(\alpha) & =\sqrt{\frac{2}{\pi}} \int_{0}^{\frac{1}{2}} \mathrm{~d} t \frac{1-(1-t)^{\alpha}}{t^{3 / 2}(1-t)^{3 / 2}} \\
& =\frac{4}{\sqrt{\pi}}{ }_{2} F_{1}\left(-\frac{1}{2}, \frac{3}{2}-\alpha ; \frac{1}{2} ; \frac{1}{2}\right), \\
C_{2}(\alpha) & =\sqrt{\frac{2}{\pi}} \int_{0}^{\frac{1}{2}} \frac{t^{\alpha}}{t^{3 / 2}(1-t)^{3 / 2}} \\
& =\sqrt{\frac{2}{\pi}} B_{1 / 2}\left(\alpha-\frac{1}{2},-\frac{1}{2}\right),
\end{aligned}
$$

and

$$
\begin{aligned}
C_{3}(\alpha)= & \frac{1}{\pi} \int_{0}^{\frac{1}{2}} \mathrm{~d} t_{1} \int_{0}^{\frac{1}{2}} \mathrm{~d} t_{2} \\
& \times \frac{1+\left(1-t_{1}-t_{2}\right)^{\alpha}-\left(1-t_{1}\right)^{\alpha}-\left(1-t_{2}\right)^{\alpha}}{t_{1}^{3 / 2}\left(1-t_{1}\right)^{3 / 2} t_{2}^{3 / 2}\left(1-t_{2}\right)^{3 / 2}},
\end{aligned}
$$

which takes the coupling (15) into account.

\section{Appendix C: Matrix element distribution for product structure}

In this appendix we derive the distribution of the matrix elements (14) for the case that the coupling matrix $V_{12}$ has the product structure $V_{12}=V_{1} V_{2}$ with $V_{\ell}$ only acting on the $\ell$-th subsystem. This situation for example occurs for the coupling (17) of the random matrix transition ensemble with product phases and the coupling (27) of the coupled kicked tops. As defined in Sec. II B 2, we have

$$
\tilde{v}^{2} \omega_{j k}=\left|\left\langle j k\left|V_{12}\right| j^{\prime} k^{\prime}\right\rangle\right|^{2}=\left|\left\langle j k\left|V_{1} V_{2}\right| j^{\prime} k^{\prime}\right\rangle\right|^{2},
$$

where in the second equality the product structure of $V_{12}$ has been used. As $|j k\rangle$ and $\left|j^{\prime} k^{\prime}\right\rangle$ are eigenstates of the uncoupled system, one can write

$$
\begin{aligned}
\tilde{v}^{2} \omega_{j k} & =\left|\left\langle j k\left|V_{1}\right| j^{\prime} k^{\prime}\right\rangle\right|^{2}\left|\left\langle j k\left|V_{2}\right| j^{\prime} k^{\prime}\right\rangle\right|^{2} \\
& ={\tilde{v_{1}}}^{2} \omega_{1_{j k}}{\tilde{v_{2}}}^{2} \omega_{2_{j k}}=\tilde{v}^{2} \omega_{1_{j k}} \omega_{2_{j k}},
\end{aligned}
$$

which defines $\omega_{1_{j k}}$ and $\omega_{2_{j k}}$. In the COE case both $\omega_{1_{j k}}$ and $\omega_{2_{j k}}$ follow the Porter-Thomas distribution (15), while for the CUE both obey the exponential (16). Thus, for the distribution $\rho_{V_{12}}(\omega)$ of the matrix elements of $V_{12}$ one gets for the $\mathrm{COE}$

$$
\begin{aligned}
\rho_{V_{12}}(\omega) & =\sum_{j^{\prime} k^{\prime} \neq j k} \delta\left(\omega-\omega_{j^{\prime} k^{\prime}}\right) \\
& =\sum_{j^{\prime} k^{\prime} \neq j k} \delta\left(\omega-\omega_{1_{j^{\prime} k^{\prime}}} \omega_{2_{j^{\prime} k^{\prime}}}\right) \\
& =\int \mathrm{d} \omega_{1} \mathrm{~d} \omega_{2} \delta\left(\omega-\omega_{1} \omega_{2}\right) \frac{\mathrm{e}^{-\left(\omega_{1}+\omega_{2}\right) / 2}}{2 \pi \sqrt{\omega_{1} \omega_{2}}} \\
& =\int \mathrm{d} \omega_{2} \frac{\mathrm{e}^{-\left(\frac{\omega}{\omega_{2}}+\omega_{2}\right) / 2}}{2 \pi \omega_{2} \sqrt{\omega}} \\
& =\frac{1}{\pi \sqrt{\omega}} K_{0}(\sqrt{\omega}) .
\end{aligned}
$$

Here $K_{\nu}$ is the modified Bessel function of the second kind [66. Eq. 10.25.3]. Similarly, one gets for the CUE case

$$
\rho_{V_{12}}(\omega)=2 K_{0}(2 \sqrt{\omega}) \text {. }
$$

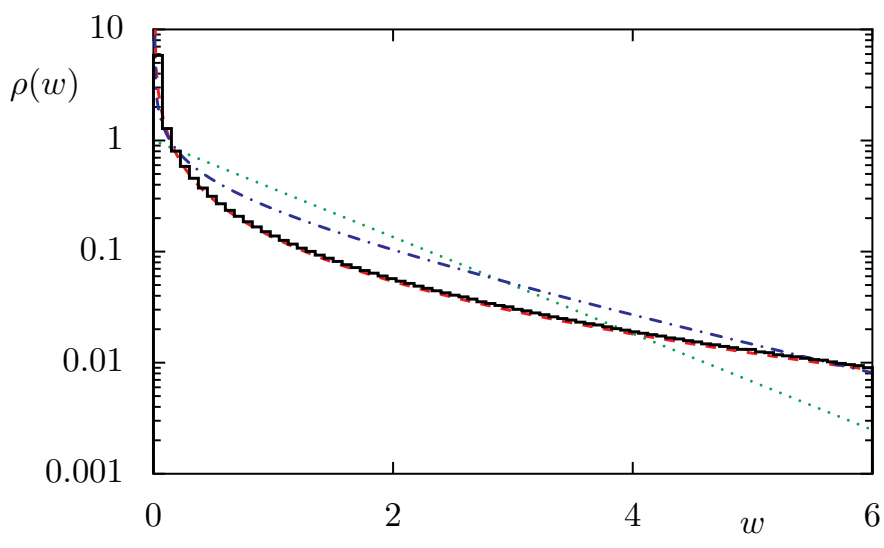

FIG. 9. Matrix element distribution for the coupled kicked tops in comparison with the prediction (C1), red dashed line, and exponential distribution (16), green dotted line, and the Porter-Thomas distribution (15), blue dash-dotted line. 
Figure 9 shows the matrix element distribution for the coupled kicked tops in comparison with the prediction (C1). Very good agreement is found, while the random matrix transition ensemble (8) with coupling (11) gives in the CUE case the exponential distribution 16 and in the COE case the PorterThomas distribution (15).
[1] A. Einstein, B. Podolsky, and N. Rosen, Can quantummechanical description of physical reality be considered complete?, Phys. Rev. 47, 777 (1935).

[2] R. Horodecki, P. Horodecki, M. Horodecki, and K. Horodecki, Quantum entanglement, Rev. Mod. Phys. 81, 865 (2009).

[3] M. A. Nielsen and I. L. Chuang, Quantum Computation and Quantum Information, Cambridge University Press, Cambridge (2010).

[4] S. Debnath, N. M. Linke, C. Figgatt, K. A. Landsman, K. Wright, and C. Monroe, Demonstration of a small programmable quantum computer with atomic qubits, Nature 536, 63 (2016).

[5] L. Amico, R. Fazio, A. Osterloh, and V. Vedral, Entanglement in many-body systems, Rev. Mod. Phys. 80, 517 (2008).

[6] A. Polkovnikov, K. Sengupta, A. Silva, and M. Vengalattore, Colloquium: Nonequilibrium dynamics of closed interacting quantum systems, Rev. Mod. Phys. 83, 863 (2011).

[7] F. Borgonovi, F. M. Izrailev, L. F. Santos, and V. G. Zelevinsky, Quantum chaos and thermalization in isolated systems of interacting particles, Phys. Rep. 626, 1 (2016).

[8] L. D'Alessio, Y. Kafri, A. Polkovnikov, and M. Rigol, From quantum chaos and eigenstate thermalization to statistical mechanics and thermodynamics, Adv. Phys. 65, 239 (2016).

[9] S. A. Parameswaran, A. C. Potter, and R. Vasseur, Eigenstate phase transitions and the emergence of universal dynamics in highly excited states, Ann. Phys. 529, 1600302 (2017).

[10] D. A. Abanin and Z. Papić, Recent progress in many-body localization, Ann. Phys. (Berlin) 529, 1700169 (2017).

[11] D. J. Luitz and Y. Bar Lev, The ergodic side of the many-body localization transition, Ann. Phys. 529, 1600350 (2017).

[12] J. von Neumann, Mathematische Grundlagen der Quantenmechanik, Julius Springer, Berlin (1932).

[13] A. Rényi, On measures of entropy and information, in J. Neyman (editor) "Proceedings of the Fourth Berkeley Symposium on Mathematical Statistics and Probability, Volume 1: Contributions to the Theory of Statistics", 547, Univ. of Calif. Press, Berkeley, Calif. (1961), pp. 547.

[14] J. Havrda and F. Charvát, Quantification method of classification processes. Concept of structural a-entropy, Kybernetika $\mathbf{3}$, 30 (1967).

[15] C. Tsallis, Possible generalization of Boltzmann-Gibbs statistics, J. Stat. Phys. 52, 479 (1988).

[16] I. Bengtsson and K. Życzkowski, Geometry of Quantum States: An Introduction to Quantum Entanglement, Cambridge University Press, Cambridge, England, 1st edition (2006).

[17] V. A. Marčenko and L. A. Pastur, Distribution of eigenvalues for some sets of random matrices, Math. USSR Sbornik 1, 457 (1967).

[18] H.-J. Sommers and K. Życzkowski, Statistical properties of random density matrices, J. Phys. A 37, 8457 (2004).

[19] E. Lubkin, Entropy of an $n$-system from its correlation with a $k$-reservoir, J. Math. Phys. 19, 1028 (1978).

[20] D. N. Page, Average entropy of a subsystem, Phys. Rev. Lett. 71, 1291 (1993).

[21] S. Sen, Average entropy of a quantum subsystem, Phys. Rev. Lett. 77, 1 (1996).
[22] A. Lakshminarayan, Entangling power of quantized chaotic systems, Phys. Rev. E 64, 036207 (2001).

[23] P. A. Miller and S. Sarkar, Entropy production, dynamical localization and criteria for quantum chaos in the open quantum kicked rotor, Nonlinearity 12, 419 (1999).

[24] J. N. Bandyopadhyay and A. Lakshminarayan, Testing statistical bounds on entanglement using quantum chaos, Phys. Rev. Lett. 89, 060402 (2002).

[25] J. N. Bandyopadhyay and A. Lakshminarayan, Entanglement production in coupled chaotic systems: Case of the kicked tops, Phys. Rev. E 69, 016201 (2004).

[26] S. Khlebnikov and M. Kruczenski, Locality, entanglement, and thermalization of isolated quantum systems, Phys. Rev. E 90 , 050101 (2014).

[27] W. Beugeling, A. Andreanov, and M. Haque, Global characteristics of all eigenstates of local many-body Hamiltonians: participation ratio and entanglement entropy, J. Stat. Mech. 2015, P02002 (2015).

[28] P. Ponte, Z. Papić, F. Huveneers, and D. A. Abanin, Many-body localization in periodically driven systems, Phys. Rev. Lett. 114, 140401 (2015).

[29] J. R. Garrison and T. Grover, Does a single eigenstate encode the full Hamiltonian?, Phys. Rev. X 8, 021026 (2018).

[30] W. Beugeling, A. Bäcker, R. Moessner, and M. Haque, Statistical properties of eigenstate amplitudes in complex quantum systems, Phys. Rev. E 98, 022204 (2018).

[31] L. Hackl, L. Vidmar, M. Rigol, and E. Bianchi, Average eigenstate entanglement entropy of the $x y$ chain in a transverse field and its universality for translationally invariant quadratic Fermionic models, Phys. Rev. B 99, 075123 (2019).

[32] S. C. L. Srivastava, S. Tomsovic, A. Lakshminarayan, R. Ketzmerick, and A. Bäcker, Universal scaling of spectral fluctuation transitions for interacting chaotic systems, Phys. Rev. Lett. 116, 054101 (2016).

[33] A. Lakshminarayan, S. C. L. Srivastava, R. Ketzmerick, A. Bäcker, and S. Tomsovic, Entanglement and localization transitions in eigenstates of interacting chaotic systems, Phys. Rev. E 94, 010205(R) (2016).

[34] S. Tomsovic, A. Lakshminarayan, S. C. L. Srivastava, and A. Bäcker, Eigenstate entanglement between quantum chaotic subsystems: Universal transitions and power laws in the entanglement spectrum, Phys. Rev. E 98, 032209 (2018).

[35] J. J. Pulikkottil, A. Lakshminarayan, S. C. L. Srivastava, A. Bäcker, and S. Tomsovic, Entanglement production by interaction quenches of quantum chaotic subsystems, arXiv:1909.04733 [quant-ph] (2019).

[36] F. Haake, M. Kuś, and R. Scharf, Classical and quantum chaos for a kicked top, Z. Phys. B 65, 381 (1987).

[37] G. M. D’Ariano, L. R. Evangelista, and M. Saraceno, Classical and quantum structures in the kicked-top model, Phys. Rev. A 45, 3646 (1992).

[38] F. Haake, Quantum Signatures of Chaos, Springer-Verlag, Berlin, 3rd revised and enlarged edition (2010).

[39] P. A. Miller and S. Sarkar, Signatures of chaos in the entanglement of two coupled quantum kicked tops, Phys. Rev. E 60, 1542 (1999). 
[40] A. Tanaka, H. Fujisaki, and T. Miyadera, Saturation of the production of quantum entanglement between weakly coupled mapping systems in a strongly chaotic region, Phys. Rev. E 66, 045201 (2002).

[41] H. Fujisaki, T. Miyadera, and A. Tanaka, Dynamical aspects of quantum entanglement for weakly coupled kicked tops, Phys. Rev. E 67, 066201 (2003).

[42] M. Žnidarič and T. Prosen, Fidelity and purity decay in weakly coupled composite systems, J. Phys. A 36, 2463 (2003).

[43] R. Demkowicz-Dobrzański and M. Kuś, Global entangling properties of the coupled kicked tops, Phys. Rev. E 70, 066216 (2004).

[44] C. M. Trail, V. Madhok, and I. H. Deutsch, Entanglement and the generation of random states in the quantum chaotic dynamics of kicked coupled tops, Phys. Rev. E 78, 046211 (2008).

[45] H. Kubotani, S. Adachi, and M. Toda, Exact formula of the distribution of Schmidt eigenvalues for dynamical formation of entanglement in quantum chaos, Phys. Rev. Lett. 100, 240501 (2008).

[46] H. Kubotani, S. Adachi, and M. Toda, Measuring dynamical randomness of quantum chaos by statistics of Schmidt eigenvalues, Phys. Rev. E 87, 062921 (2013).

[47] Z. Puchała, Ł. Pawela, and K. Życzkowski, Distinguishability of generic quantum states, Phys. Rev. A 93, 062112 (2016).

[48] S. Kumar, B. Sambasivam, and S. Anand, Smallest eigenvalue density for regular or fixed-trace complex Wishart-Laguerre ensemble and entanglement in coupled kicked tops, J. Phys. A 50, 345201 (2017).

[49] S. Adachi, H. Kubotani, and M. Toda, Exact distribution of largest Schmidt eigenvalue for quantum entanglement, J. Phys. A 52, 405304 (2019).

[50] S. Chaudhury, A. Smith, B. E. Anderson, S. Ghose, and P. S. Jessen, Quantum signatures of chaos in a kicked top, Nature 461, 768 (2009).

[51] C. Neill, P. Roushan, M. Fang, Y. Chen, M. Kolodrubetz, Z. Chen, A. Megrant, R. Barends, B. Campbell, B. Chiaro, A. Dunsworth, E. Jeffrey, J. Kelly, J. Mutus, P. J. J. O’Malley, C. Quintana, D. Sank, A. Vainsencher, J. Wenner, T. C. White, A. Polkovnikov, and J. M. Martinis, Ergodic dynamics and thermalization in an isolated quantum system, Nature Physics 12, 1037 (2016)

[52] E. J. Meier, J. Ang'ong'a, F. A. An, and B. Gadway, Exploring quantum signatures of chaos on a Floquet synthetic lattice, Phys. Rev. A 100, 013623 (2019).

[53] V. R. Krithika, V. S. Anjusha, U. T. Bhosale, and T. S. Mahesh, NMR studies of quantum chaos in a two-qubit kicked top, Phys. Rev. E 99, 032219 (2019).

[54] M. H. Muñoz-Arias, P. M. Poggi, P. S. Jessen, and I. H. Deutsch, Simulating nonlinear dynamics of collective spins via quantum measurement and feedback, arXiv:1907.12606 [quantph] (2019).

[55] G. J. Milburn, Simulating nonlinear spin models in an ion trap, arXiv:quant-ph/9908037(1999).

[56] X. Wang, S. Ghose, B. C. Sanders, and B. Hu, Entanglement as a signature of quantum chaos, Phys. Rev. E 70, 016217 (2004).

[57] S. Dogra, V. Madhok, and A. Lakshminarayan, Quantum signatures of chaos, thermalization, and tunneling in the exactly solvable few-body kicked top, Phys. Rev. E 99, 062217 (2019).
[58] L. M. Sieberer, T. Olsacher, A. Elben, M. Heyl, P. Hauke, F. Haake, and P. Zoller, Digital quantum simulation, trotter errors, and quantum chaos of the kicked top, npj Quantum Information 5 (2019).

[59] P. Zanardi, C. Zalka, and L. Faoro, Entangling power of quantum evolutions, Phys. Rev. A 62, 030301(R) (2000).

[60] R. Pal and A. Lakshminarayan, Entangling power of timeevolution operators in integrable and nonintegrable many-body systems, Phys. Rev. B 98, 174304 (2018).

[61] A. Pandey and M. L. Mehta, Gaussian ensembles of random Hermitian matrices intermediate between orthogonal and unitary ones, Commun. Math. Phys. 87, 449 (1983).

[62] J. B. French, V. K. B. Kota, A. Pandey, and S. Tomsovic, Statistical properties of many-particle spectra $V$. Fluctuations and symmetries, Ann. Phys. (N.Y.) 181, 198 (1988).

[63] M. L. Mehta, Random Matrices, Elsevier, 3rd edition (2004).

[64] A. Lakshminarayan, Z. Puchała, and K. Życzkowski, Diagonal unitary entangling gates and contradiagonal quantum states, Phys. Rev. A 90, 032303 (2014).

[65] C. E. Porter and R. G. Thomas, Fluctuations of nuclear reaction widths, Phys. Rev. 104, 483 (1956).

[66] NIST Digital library of mathematical functions, http://dlmf.nist.gov/, Release 1.0 .23 of 2019-06-15, F. W. J. Olver, A. B. Olde Daalhuis, D. W. Lozier, B. I. Schneider, R. F. Boisvert, C. W. Clark, B. R. Miller and B. V. Saunders, (eds.).

[67] O. Bohigas, M. J. Giannoni, and C. Schmit, Characterization of chaotic quantum spectra and universality of level fluctuation laws, Phys. Rev. Lett. 52, 1 (1984).

[68] T. Tkocz, M. Smaczyński, M. Kuś, O. Zeitouni, and K. Życzkowski, Tensor products of random unitary matrices, Random Matrices: Theory Appl. 1, 1250009 (2012).

[69] S. Tomsovic, Bounds on the Time-Reversal Noninvariant Nucleon-Nucleon Interaction Derived from Transition Strength Fluctuations, Ph.D. thesis, University of Rochester (1986), [UR Report No. 974, 1987].

[70] A. Y. Abul-Magd, Level statistics for nearly integrable systems, Phys. Rev. E 80, 017201 (2009).

[71] S. K. Foong and S. Kanno, Proof of Page's conjecture on the average entropy of a subsystem, Phys. Rev. Lett. 72, 1148 (1994).

[72] J. Sánchez-Ruiz, Simple proof of Page's conjecture on the average entropy of a subsystem, Phys. Rev. E 52, 5653 (1995).

[73] S. C. L. Srivastava, A. Lakshminarayan, S. Tomsovic, and A. Bäcker, Ordered level spacing probability densities, J. Phys. A 52, 025101 (2019).

[74] H. Li and F. D. M. Haldane, Entanglement spectrum as a generalization of entanglement entropy: Identification of topological order in non-Abelian fractional quantum Hall effect states, Phys. Rev. Lett. 101, 010504 (2008).

[75] C. H. Bennett, H. J. Bernstein, S. Popescu, and B. Schumacher, Concentrating partial entanglement by local operations, Phys. Rev. A 53, 2046 (1996).

[76] N. Ullah and C. E. Porter, Invariance hypothesis and Hamiltonian matrix elements correlations, Phys. Lett. 6, 301 (1963).

[77] Z. Puchała and J. A. Miszczak, Symbolic integration with respect to the Haar measure on the unitary groups, Bull. Pol. Ac.: Tech. 65, 21 (2017). 